\title{
Designing Bayesian Sampling Plans with Adaptive Progressive Hybrid Censored Samples
}

\author{
TaChen Liang \\ Wayne State University, Detroit, MI 48202, USA \\ Correspondence should be addressed to TaChen Liang; aa4156@wayne.edu
}

Received 2 May 2014; Accepted 29 September 2014; Published 16 November 2014

Academic Editor: Chin-Shang Li

Copyright (C) 2014 TaChen Liang. This is an open access article distributed under the Creative Commons Attribution License, which permits unrestricted use, distribution, and reproduction in any medium, provided the original work is properly cited.

\begin{abstract}
This paper studies the acceptance sampling for exponential distributions with type-I and type-II adaptive progressive hybrid censored samples. Algorithms are proposed for deriving Bayesian sampling plans. We compare the performance of the proposed sampling plans with the sampling plans of Lin and Huang (2012). The numerical results indicate that the proposed sampling plans outperform the sampling plans of Lin and Huang (2012).
\end{abstract}

\section{Introduction}

Suppose we are given a batch of $N$ lifetime components for acceptance sampling. We let $X_{1}, \ldots, X_{N}$ denote the lifetimes of these $N$ units. It is assumed that $X_{1}, \ldots, X_{N}$ are mutually independent and follow an exponential distribution, having expected lifetime $\theta=1 / \lambda$, and the parameter $\lambda$ follows a gamma $(\alpha, \beta)$ prior distribution. In the context of life test experiment, $n$ identical units are sampled from the batch and placed on life test without replacement with a suitable sampling scheme. At the end of the experiment, let $\tau$ denote the duration of the experiment, and let $D$ be the number of failures among the $n$ items put on life test. Let $a$ be an action regarding the acceptance sampling. When $a=1$, it means accepting the batch, while when $a=0$, it means rejecting the batch. Let $C_{s}$ denote the cost per unit inspected. Also, let $C_{r}$ be the loss of rejecting the batch and let $(1-n / N) h(\lambda)$ be the loss of accepting the batch where $h(\lambda)=a_{0}+a_{1} \lambda+a_{2} \lambda^{2}, a_{0} \geq 0$, $a_{1} \geq 0$, and $a_{2}>0$. In many situations, the cost of time used for life test is an essential issue and should be taken as a part of the loss function. We let $C_{t}$ be the cost per unit time used for the life test experiment. When the life test experiment terminates, the unfailed components can be reused and thus have salvage value $r_{s}$, where $C_{s}>r_{s} \geq 0$. Thus, many researchers including Chen et al. [1], Liang and Yang [2], and Lin and
Huang [3] have considered using the loss function $L$ for the acceptance sampling, where

$$
L=C_{s} n-r_{s}(n-D)+C_{t} \tau+C_{r}(1-a)+a\left(1-\frac{n}{N}\right) h(\lambda) .
$$

Lin and Huang [3] have studied acceptance sampling for exponential distributions with the loss $L$ based on adaptive type-I and type-II progressive hybrid censored samples. For the adaptive progressive hybrid censoring (APHC), a positive time $T$ and progressive censoring scheme $\left(R_{1}, \ldots, R_{m}\right)$ should be determined before the life test experiment, where $1 \leq m \leq n, R_{j} \geq 0$, for $j=1, \ldots, m$, and $R_{1}+\cdots+R_{m}+m=n$. Let $\underline{X}$ denote the observable variables obtained either by using type-I APHC or by using type-II APHC and $\underline{x}$ an observed value of $X$. A decision function $\delta$ is a function defined on the sample space of the observable variables $\underline{X}$ such that $\delta(\underline{x})$ is the probability of accepting the batch when $\underline{X}=\underline{x}$ is observed. The determination of the design parameters $\left(n, m,\left(R_{1}, \ldots, R_{m}\right), T\right)$ and the decision function $\delta$ is called a sampling plan, which is denoted by $(n, m$, $\left.\left(R_{1}, \ldots, R_{m}\right), T, \delta\right)$.

With the $L$ loss of $(1)$ and the $\Gamma(\alpha, \beta)$ prior distribution, the Bayes risk $R\left(n, m,\left(R_{1}, \ldots, R_{m}\right), T, \delta\right)$ associated with the sampling plan $\left(n, m,\left(R_{1}, \ldots, R_{m}\right), T, \delta\right)$ can be expressed as

$$
R\left(n, m,\left(R_{1}, \ldots, R_{m}\right), T, \delta\right)=S_{1}+S_{2}+S_{3}+S_{4}+S_{5},
$$




$$
\begin{aligned}
& S_{1}=E\left[C_{r}(1-\delta(\underline{X}))\right], \\
& S_{2}=E\left[\delta(\underline{X})\left(1-\frac{n}{N}\right) h(\Lambda)\right], \\
& S_{3}=n\left(C_{s}-r_{s}\right), \\
& S_{4}=r_{s} E[D \mid n, T], \\
& S_{5}=C_{t} E[\tau] .
\end{aligned}
$$

A sampling plan, say $\left(n_{B}, m_{B},\left(R_{1 B}, \ldots, R_{m_{B} B}\right), T_{B}, \delta_{B}\right)$, is said to be a Bayesian sampling plan if $\left(n_{B}, m_{B}\right.$, $\left.\left(R_{1 B}, \ldots, R_{m_{B} B}\right), T_{B}, \delta_{B}\right)$ minimizes the Bayes risks $R(n, m$, $\left.\left(R_{1}, \ldots, R_{m}\right), T, \delta\right)$ among all sampling plans $\left(n, m,\left(R_{1}, \ldots\right.\right.$, $\left.\left.R_{m}\right), T, \delta\right)$. Lin and Huang [3] claimed having derived the Bayesian sampling plan for each type of APHC.

However, the reported sampling plans are not the Bayesian sampling plan since the associated Bayes risks are much larger than the minimum Bayes risks.

The goal of this paper is to find the Bayesian sampling plan for the acceptance sampling with the loss $L$. The paper is organized as follows. Section 2 deals with the derivation of the Bayesian sampling plan with type-I APHC samples. For the given parameters $\left(n, m,\left(R_{1}, \ldots, R_{m}\right), T\right)$, a Bayes decision function $\delta_{B 1}$ is derived. Then, Algorithm $\mathrm{A}$ is proposed for deriving the Bayesian sampling plan $\mathrm{BSP}_{1} \equiv\left(n_{B 1}, m_{B 1}\right.$, $\left.\left(R_{1, B 1}, \ldots, R_{m_{B 1}, B 1}\right), T_{B 1}, \delta_{B 1}\right)$. Section 3 deals with the derivation of the Bayesian sampling plan with type-II APHC samples. For the given parameters $\left(n, m,\left(R_{1}, \ldots, R_{m}\right), T\right)$, a Bayes decision function $\delta_{B 2}$ is derived. Then, Algorithm B is proposed for deriving the Bayesian sampling plan $\mathrm{BSP}_{2} \equiv$ $\left(n_{B 2}, m_{B 2},\left(R_{1, B 2}, \ldots, R_{m_{B 2}, B 2}\right), T_{B 2}, \delta_{B 2}\right)$. In Section 4 , we compare the performance of the proposed sampling plans with the sampling plans of Lin and Huang [3]. The numerical results indicate that the proposed sampling plans perform better than that of Lin and Huang [3].

\section{Derivation of the Bayesian Sampling Plan BSP}

For type-I APHC, during the experiment, for each $j=1, \ldots$, $m-1$, when the $j$ th failure is observed, immediately after the failure, $R_{j}$ functioning items are randomly removed from the life test. Let $Y_{j: m, n}$ denote the lifetime of the $j$ th failed item. Also, let $Y_{m: m, n}$ denote the lifetime of the $m$ th failed item. When $Y_{m: m, n} \geq T$, the experiment terminates at time $T$. When $Y_{m: m, n}<T$, at the $m$ th failure, do not follow the prespecified censoring scheme to remove the remaining $R_{m}$ functioning items; instead, continue to observe failures without any further withdrawals up to time $T$. In either case, let $D$ denote the number of failures before time $T$. Thus, $0 \leq D \leq$ $n-\left(R_{1}+\cdots+R_{m-1}\right)$. We denote the lifetimes of the failures after the $m$ th failure by $Y_{j: m, n}, m<j \leq n-\left(R_{1}+\cdots+R_{m-1}\right)$. Let $\underline{Y}(D)=\left(Y_{1: m, n}, \ldots, Y_{D: m, n}\right), 1 \leq D \leq n-\left(R_{1}+\cdots+R_{m-1}\right)$. Let $(d, y(d))=\left(d, y_{1}, \ldots, y_{d}\right)$ be observed values of $(D, \underline{Y}(D))$. Let $\bar{n}_{d}=R_{1}+\cdots+R_{d}, 1 \leq d \leq m-1$, and $n_{0} \equiv 0$. The duration of the life test experiment is $\tau=\min \left(Y_{n-n_{m-1}: n}, T\right)$. The observable variables $(D, \underline{Y}(D))$ have a joint probability density function $f_{1}(d, y(d) \mid \bar{\lambda})$ given as follows:

$$
f_{1}(d, \underline{y}(d) \mid \lambda)=A_{1}(n, m, d) \lambda^{d} e^{-\lambda y_{1}(n, m, d, T)},
$$

where $y_{1}(n, m, d, T)$ is an observed value of $Y_{1}(n, m, D, T)$ and $Y_{1}(n, m, D, T)$

$$
= \begin{cases}n T, & \text { if } D=0 \\ \sum_{i=1}^{D}\left(R_{i}+1\right) Y_{i: m, n} & \\ +\left(n-D-n_{D}\right) T, & \text { if } 1 \leq D \leq m-1 ; \\ \sum_{i=1}^{m-1}\left(R_{i}+1\right) Y_{i: m, n}+\sum_{j=m}^{D} Y_{j: m, n} & \\ +\left(n-D-n_{m-1}\right) T, & \text { if } m \leq D \leq n-n_{m-1},\end{cases}
$$

where $A_{1}(n, m, d)=\prod_{j=1}^{d} \Delta_{j}$ and $\Delta_{j}=n-(j-1)-\sum_{l=1}^{j-1} R_{l}$, $j=1, \ldots, m-1$, and $\Delta_{j}=n-(j-1)-\sum_{l=1}^{m-1} R_{l}$ for $j \geq m$.

2.1. A Bayes Decision Function $\delta_{B 1}$. Let $g(\lambda)$ denote the probability density of a $\Gamma(\alpha, \beta)$ prior distribution. Let

$$
\begin{aligned}
f_{1}(d, \underline{y}(d)) & =\int f_{1}(d, \underline{y}(d) \mid \lambda) g(\lambda) d \lambda, \\
g_{1}(\lambda \mid d, \underline{y}(d)) & =\frac{f_{1}(d, \underline{y}(d) \mid \lambda) g(\lambda)}{f_{1}(d, \underline{y}(d))}, \\
\varphi_{1}(d, \underline{y}(d)) & =\left(1-\frac{n}{N}\right) \int h(\lambda) g_{1}(\lambda \mid d, \underline{y}(d)) d \lambda .
\end{aligned}
$$

Note that $f_{1}(d, y(d))$ is the marginal joint probability density of $(D, \underline{Y}(D)), \overline{g_{1}}(\lambda \mid d, y(d))$ is the posterior probability density of $\Lambda$, and $\varphi_{1}(d, y(\bar{d}))$ is the posterior expectation of $(1-n / N) h(\lambda)$ given $(\overline{D,} \underline{Y}(D))=(d, \underline{y}(d))$ being observed. For each observed $(d, \underline{y}(d))$, define

$$
\delta_{B 1}(d, \underline{y}(d))= \begin{cases}1 & \text { if } \varphi_{1}(d, \underline{y}(d))<C_{r} ; \\ 0, & \text { otherwise. }\end{cases}
$$

Note that

$$
\begin{aligned}
S_{1}+S_{2} & =E\left[\delta(D, \underline{Y}(D))\left(h(\Lambda)-C_{r}\right)\right]+C_{r} \\
& \equiv S\left(\delta \mid n, m,\left(R_{1}, \ldots, R_{m}\right), T\right)+C_{r} .
\end{aligned}
$$

Theorem 1. $\delta_{B 1}$ is a Bayes decision function in the sense that, for each fixed sampling parameters $\left(n, m,\left(R_{1}, \ldots, R_{m}\right), T\right)$, $S\left(\delta_{B 1} \mid n, m,\left(R_{1}, \ldots, R_{m}\right), T\right) \leq S\left(\delta \mid n, m,\left(R_{1}, \ldots, R_{m}\right), T\right)$ for all decision functions $\delta$.

Proof. Let $A=\left\{(d, y(d)) \mid \varphi_{1}(d, y(d))<C_{r}\right\}$ and $A^{-}=$ $\left\{(d, y(d)) \mid \varphi_{1}(d, y(d))>C_{r}\right\}$. 
Then,

$$
\begin{gathered}
S\left(\delta \mid n, m,\left(R_{1}, \ldots, R_{m}\right), T\right)-S\left(\delta_{B 1} \mid n, m,\left(R_{1}, \ldots, R_{m}\right), T\right) \\
=\int_{A} f_{1}(d, \underline{y}(d))\left[\delta(d, \underline{y}(d))-\delta_{B 1}(d, \underline{y}(d))\right] \\
\quad \times\left(\varphi_{1}(d, \underline{y}(d))-C_{r}\right) d(d, \underline{y}(d)) \\
+\int_{A^{-}} f_{1}(d, \underline{y}(d))\left[\delta(d, \underline{y}(d))-\delta_{B 1}(d, \underline{y}(d))\right] \\
\quad \times\left(\varphi_{1}(d, \underline{y}(d))-C_{r}\right) d(d, \underline{y}(d)) .
\end{gathered}
$$

On $A, \varphi_{1}(d, y(d))-C_{r}<0, \delta(d, y(d))-\delta_{B 1}(d, y(d))=$ $\delta(d, y(d))-\overline{1} \leq 0$. Thus,

$$
\begin{gathered}
\int_{A} f_{1}(d, \underline{y}(d))\left[\delta(d, \underline{y}(d))-\delta_{B 1}(d, \underline{y}(d))\right] \\
\times\left(\varphi_{1}(d, \underline{y}(d))-C_{r}\right) d(d, \underline{y}(d)) \geq 0 .
\end{gathered}
$$

On $A^{-}, \varphi_{1}(d, \underline{y}(d))-C_{r}>0, \delta(d, \underline{y}(d))-\delta_{B 1}(d, \underline{y}(d))=$ $\delta(d, y(d))-0 \overline{\geq}$. Thus,

$$
\begin{gathered}
\int_{A^{-}} f_{1}(d, \underline{y}(d))\left[\delta(d, \underline{y}(d))-\delta_{B 1}(d, \underline{y}(d))\right] \\
\times\left(\varphi_{1}(d, \underline{y}(d))-C_{r}\right) d(d, \underline{y}(d)) \geq 0 .
\end{gathered}
$$

Combining (9)-(11) leads to the results of the theorem.

2.1.1. An Alternative Form of $\delta_{B 1}$. A straightforward computation shows that

$\varphi_{1}(d, \underline{y}(d))$

$$
\begin{aligned}
= & \left(1-\frac{n}{N}\right) \\
& \times\left(a_{0}+\frac{a_{1}(d+\alpha)}{\left[\beta+y_{1}(n, m, d, T)\right]}+\frac{a_{2}(d+\alpha)(d+\alpha+1)}{\left[\beta+y_{1}(n, m, d, T)\right]^{2}}\right) .
\end{aligned}
$$

If $a_{0}(1-n / N) \geq C_{r}$, then $\varphi_{1}(d, y(d))>C_{r}$ for all $(d, y(d))$. Hence, $\delta_{B 1}(d, y(d))=0$ for all $(\bar{d}, y(d))$. In such a situation, we should take $n=0$ and consider the sampling plan with no sample data.

$$
\begin{aligned}
& \text { As } a_{0}(1-n / N)<C_{r}, \text { let } \\
& \begin{aligned}
B(d)= & {\left[a_{1}(d+\alpha)\right]^{2} } \\
& +4 a_{2}\left(\frac{C_{r}}{(1-n / N)}-a_{0}\right)(d+\alpha)(d+\alpha+1) .
\end{aligned}
\end{aligned}
$$

Then,

$$
\begin{aligned}
& \delta_{B 1}(d, \underline{y}(d))=1 \\
& \text { iff } a_{0}+\frac{a_{1}(d+\alpha)}{\left[\beta+y_{1}(n, m, d, T)\right]}+\frac{a_{2}(d+\alpha)(d+\alpha+1)}{\left[\beta+y_{1}(n, m, d, T)\right]^{2}} \\
& \quad<\frac{C_{r}}{(1-n / N)} \\
& \text { iff } y_{1}(n, m, d, T)>\left(\frac{a_{1}(d+\alpha)+\sqrt{B(d)}}{2\left(C_{r} /(1-n / N)-a_{0}\right)}-\beta\right) \vee 0 \\
& \quad \equiv c(d) .
\end{aligned}
$$

Note that $c(d)$ is increasing in $d$ for $d=0, \ldots, m, \ldots, n-n_{m-1}$. Also, $y_{1}(n, m, d, T) \leq n T$.

If $n T \leq c(0)$, then $y_{1}(n, m, d, T) \leq c(d)$ for all $y_{1}(n, m, d, T)$. Thus, $\delta_{B 1}(d, y(d)) \equiv 0$ for all $y_{1}(n, m, d, T)$. So, we request $n T>c(0)$.

2.1.2. Bayes Decision Function for No Sample Data Case. For no sample situation, the associated parameters are $(n, m$, $\left.\left(R_{1}, \ldots, R_{m}\right), T\right)=(0,0,(0), 0)$. is

Denote $E h=\int h(\lambda) g(\lambda) d \lambda$. The Bayes decision function

$$
\delta_{B 1}= \begin{cases}1, & \text { if } E h<C_{r} \\ 0, & \text { otherwise }\end{cases}
$$

Thus, for no sample data case, the Bayes risk of the sampling plan $\left(0,0,(0), 0, \delta_{B 1}\right)$ is $R\left(0,0,(0), 0, \delta_{B 1}\right)=\min \left(E h, C_{r}\right)$.

2.2. Derivation of Bayesian Sampling Plans. From (2), we have

$$
\begin{aligned}
R(n, m, & \left.\left(R_{1}, \ldots, R_{m}\right), T, \delta_{B 1}\right)=S_{1}+S_{2}+S_{3}+S_{4}+S_{5}, \\
S_{1} & =E\left[C_{r}\left(1-\delta_{B 1}(D, \underline{Y}(D))\right)\right] \\
S_{2} & =E\left[\delta_{B 1}(D, \underline{Y}(D))\left(1-\frac{n}{N}\right) h(\Lambda)\right] \\
S_{3} & =n\left(C_{s}-r_{s}\right) \\
S_{4} & =r_{s} E[D \mid n, T], \\
S_{5} & =C_{t} E\left[\min \left(Y_{n-n_{m-1}: m, n}, T\right)\right] .
\end{aligned}
$$

Note that $\min \left(Y_{n-n_{m-1}: m, n}, T\right) \geq \min \left(X_{(1): n}, T\right)$, where $X_{(1): n}$ is the first ordered statistic of the lifetime variables of the $n$ items put on life test. 
For each $n \geq 1$, the sampling plan $\left(n, n,(n \times 0), \infty, \delta_{B 1}\right)$ can collect as much information from the sample as the sampling plan $\left(n, m,\left(R_{1}, \ldots, R_{m}\right), T, \delta_{B 1}\right)$ does. Thus, we have

$$
\begin{aligned}
S_{1}\left(\delta_{B 1} \mid n, m,\left(R_{1}, \ldots, R_{m}\right), T\right) \\
\quad+S_{2}\left(\delta_{B 1} \mid n, m,\left(R_{1}, \ldots, R_{m}\right), T\right) \\
\geq S_{1}\left(\delta_{B 1} \mid n, n,(n \times 0), \infty\right)+S_{2}\left(\delta_{B 1} \mid n, n,(n \times 0), \infty\right) .
\end{aligned}
$$

Therefore, for any sampling plan $\left(n, m,\left(R_{1}, \ldots, R_{m}\right), T, \delta_{B 1}\right)$, the following inequality holds:

$$
\begin{aligned}
R\left(n, m,\left(R_{1}, \ldots, R_{m}\right), T, \delta_{B 1}\right) \\
\geq S^{1,2}\left(\delta_{B 1} \mid n, n,(n \times 0), \infty\right)+n\left(C_{s}-r_{s}\right) \\
\quad+C_{t} E\left[\min \left(X_{(1): n}, T\right)\right],
\end{aligned}
$$

where $S^{1,2}\left(\delta_{B 1} \mid n, n,(n \times 0), \infty\right)=S_{1}\left(\delta_{B 1} \mid n, n,(n \times 0), \infty\right)+$ $S_{2}\left(\delta_{B 1} \mid n, n,(n \times 0), \infty\right)$. So, if

$$
\begin{aligned}
S^{1,2}( & \left.\delta_{B 1} \mid n, n,(n \times 0), \infty\right)+n\left(C_{s}-r_{s}\right) \\
& +C_{t} E\left[\min \left(X_{(1): n}, T\right)\right] \geq \min \left(E h, C_{r}\right),
\end{aligned}
$$

then $\left(n, m,\left(R_{1}, \ldots, R_{m}\right), T, \delta_{B 1}\right)$ is not a Bayesian sampling plan. With this property, we propose Algorithm A for deriving a Bayesian sampling plan as follows.

Algorithm A. Define $N^{*}=\left\langle\min \left(C_{r}, E h\right) /\left(C_{s}-r_{s}\right)\right\rangle$, where $\langle x\rangle$ denotes the largest integer less than $x$.

Step 1. For each $n$ satisfying $1 \leq n \leq N^{*}$, construct all type-I adaptive progressive hybrid censoring schemes ( $n, m$, $\left.\left(R_{1}, \ldots, R_{m}\right), T\right)$ with $n T \geq c(0)$.

Step 2. For each fixed sampling scheme $\left(n, m,\left(R_{1}, \ldots\right.\right.$, $\left.\left.R_{m}\right), T\right)$, derive the Bayes decision function $\delta_{B 1} \equiv \delta_{B 1}(n, m$, $\left.\left(R_{1}, \ldots, R_{m}\right), T\right)$. Note that if

$$
\begin{aligned}
& S^{1,2}\left(\delta_{B 1} \mid n, n,(n \times 0), \infty\right)+n\left(C_{s}-r_{s}\right) \\
& \quad+C_{t} E\left[\min \left(X_{(1): n}, \frac{c(0)}{n}\right)\right] \geq \min \left(E h, C_{r}\right),
\end{aligned}
$$

then $\left(n, m,\left(R_{1}, \ldots, R_{m}\right), T, \delta_{B 1}\right)$ is not a Bayesian sampling plan.

If $R\left(n, m,\left(R_{1}, \ldots, R_{m}\right), T, \delta_{B 1}\right)>\min \left(E h, C_{r}\right)$ for all $\left(n, m,\left(R_{1}, \ldots, R_{m}\right), T, \delta_{B 1}\right)$, take $n_{B 1}=0$ and adopt the sampling plan $\left(0,0,(0), 0, \delta_{B 1}\right)$. Go to Step 8 .

Otherwise, go to Step 3.

Step 3. For each fixed $\left(n, m,\left(R_{1}, \ldots, R_{m}\right)\right)$, find the time $T_{B 1} \geq$ $c(0)$ such that

$$
\begin{aligned}
R & \left(n, m,\left(R_{1}, \ldots, R_{m}\right), T_{B 1}, \delta_{B 1}\right) \\
\quad & =\inf _{T \geq c(0)}\left\{R\left(n, m,\left(R_{1}, \ldots, R_{m}\right), T, \delta_{B 1}\right)\right\} .
\end{aligned}
$$

Step 4. For each fixed $(n, m)$, find the censoring scheme $\left(R_{B 1}, \ldots, R_{B m}\right)$ such that

$$
\begin{aligned}
R & \left(n, m,\left(R_{B 1}, \ldots, R_{B m}\right), T_{B 1}, \delta_{B 1}\right) \\
& =\inf _{\left(R_{1}, \ldots, R_{m}\right)}\left\{R\left(n, m,\left(R_{1}, \ldots, R_{m}\right), T_{B 1}, \delta_{B 1}\right)\right\} .
\end{aligned}
$$

Step 5. For each $n$, find the $m_{B 1}$ such that

$$
\begin{aligned}
R & \left(n, m_{B 1},\left(R_{B 1}, \ldots, R_{B m_{B 1}}\right), T_{B 1}, \delta_{B 1}\right) \\
& =\min _{1 \leq m \leq n} R\left(n, m,\left(R_{B 1}, \ldots, R_{B m}\right), T_{B 1}, \delta_{B 1}\right) .
\end{aligned}
$$

Step 6. Find $n_{B 1}, 0 \leq n_{B 1} \leq N^{*}$, such that

$$
\begin{aligned}
R & \left(n_{B 1}, m_{B 1},\left(R_{B 1}, \ldots, R_{B m_{B 1}}\right), T_{B 1}, \delta_{B 1}\right) \\
& =\min _{1 \leq n \leq N^{*}} R\left(n, m_{B 1},\left(R_{B 1}, \ldots, R_{B m_{B 1}}\right), T_{B 1}, \delta_{B 1}\right) .
\end{aligned}
$$

Step 7. If $R\left(n_{B 1}, m_{B 1},\left(R_{B 1}, \ldots, R_{B m_{B 1}}\right), T_{B 1}, \delta_{B 1}\right)<R(0,0,(0)$, $\left.0, \delta_{B 1}\right)$, then propose the sampling plan $\left(n_{B 1}, m_{B 1},\left(R_{B 1}, \ldots\right.\right.$, $\left.\left.R_{B m_{B 1}}\right), T_{B 1}, \delta_{B 1}\right)$; otherwise, $\left(0,0,(0), 0, \delta_{B 1}\right)$ is the proposed sampling plan.

Step 8. When $n_{B 1}=0$, we let $m_{B 1}=0,\left(R_{B 1}, \ldots, R_{B m_{B 1}}\right)=(0)$, $T_{B 1}=0$, and $\left(0,0,(0), 0, \delta_{B 1}\right)$ is the proposed sampling plan.

Theorem 2. $\left(n_{B 1}, m_{B 1},\left(R_{B 1}, \ldots, R_{B m_{B 1}}\right), T_{B 1}, \delta_{B 1}\right)$ is an optimal sampling plan in the sense that $R\left(n_{B 1}, m_{B 1},\left(R_{B 1}, \ldots\right.\right.$, $\left.\left.R_{B m_{B 1}}\right), T_{B 1}, \delta_{B 1}\right) \leq R\left(n, m,\left(R_{1}, \ldots, R_{m}\right), T, \delta\right)$ for all sampling plans $\left(n, m,\left(R_{1}, \ldots, R_{m}\right), T, \delta\right)$.

Proof. It suffices to show that, for any sampling plan $(n, m$, $\left.\left(R_{1}, \ldots, R_{m}\right), T, \delta\right)$ with $1 \leq n \leq N^{*}$, the following inequality holds:

$$
\begin{aligned}
R(n, m, & \left.\left(R_{1}, \ldots, R_{m}\right), T, \delta\right) \\
- & R\left(n_{B 1}, m_{B 1},\left(R_{B 1}, \ldots, R_{B m_{B 1}}\right), T_{B 1}, \delta_{B 1}\right) \\
= & R\left(n, m,\left(R_{1}, \ldots, R_{m}\right), T, \delta\right) \\
- & \left.R\left(n, m,\left(R_{1}, \ldots, R_{m}\right), T, \delta_{B 1}\right)\right] \\
+ & {\left[R\left(n, m,\left(R_{1}, \ldots, R_{m}\right), T, \delta_{B 1}\right)\right.} \\
& \left.-R\left(n, m,\left(R_{1}, \ldots, R_{m}\right), T_{B 1}, \delta_{B 1}\right)\right] \\
+ & {\left[R\left(n, m,\left(R_{1}, \ldots, R_{m}\right), T_{B 1}, \delta_{B 1}\right)\right.} \\
& \left.-R\left(n, m,\left(R_{B 1}, \ldots, R_{B m}\right), T_{B 1}, \delta_{B 1}\right)\right] \\
+ & {\left[R\left(n, m,\left(R_{B 1}, \ldots, R_{B m}\right), T_{B 1}, \delta_{B 1}\right)\right.} \\
& \left.-R\left(n, m_{B 1},\left(R_{B 1}, \ldots, R_{B m_{B 1}}\right), T_{B 1}, \delta_{B 1}\right)\right] \\
+ & {\left[R\left(n, m_{B 1},\left(R_{B 1}, \ldots, R_{B m_{B 1}}\right), T_{B 1}, \delta_{B 1}\right)\right.} \\
& \left.-R\left(n_{B 1}, m_{B 1},\left(R_{B 1}, \ldots, R_{B m_{B 1}}\right), T_{B 1}, \delta_{B 1}\right)\right] .
\end{aligned}
$$


By Step $2, R\left(n, m,\left(R_{1}, \ldots, R_{m}\right), T, \delta\right)-R\left(n, m,\left(R_{1}, \ldots\right.\right.$, $\left.\left.R_{m}\right), T, \delta_{B 1}\right) \geq 0$;

by Step $3, R\left(n, m,\left(R_{1}, \ldots, R_{m}\right), T, \delta_{B 1}\right)-R\left(n, m,\left(R_{1}\right.\right.$, $\left.\left.\ldots, R_{m}\right), T_{B 1}, \delta_{B 1}\right) \geq 0$;

by Step $4, R\left(n, m,\left(R_{1}, \ldots, R_{m}\right), T_{B 1}, \delta_{B 1}\right)-R(n, m$, $\left.\left(R_{B 1}, \ldots, R_{B m}\right), T_{B 1}, \delta_{B 1}\right) \geq 0$;

by Step $5, R\left(n, m,\left(R_{B 1}, \ldots, R_{B m}\right), T_{B 1}, \delta_{B 1}\right)-R\left(n, m_{B 1}\right.$, $\left.\left(R_{B 1}, \ldots, R_{B m_{B 1}}\right), T_{B 1}, \delta_{B 1}\right) \geq 0$;

by Step $6, R\left(n, m_{B 1},\left(R_{B 1}, \ldots, R_{B m_{B 1}}\right), T_{B 1}, \delta_{B 1}\right)-R\left(n_{B 1}\right.$, $\left.m_{B 1},\left(R_{B 1}, \ldots, R_{B m_{B 1}}\right), T_{B 1}, \delta_{B 1}\right) \geq 0$.

Combining the preceding inequalities and (26), we conclude this theorem.

\subsubsection{An Upper Bound of $n_{B 1}$. Since}

$$
\begin{aligned}
n_{B 1}\left(C_{s}-r_{s}\right) & \leq R\left(n_{B 1}, m_{B 1},\left(R_{B 1}, \ldots, R_{B m_{B 1}}\right), T_{B 1}, \delta_{B 1}\right) \\
& \leq R\left(0,0,(0), 0, \delta_{B 1}\right)=\min \left(E h, C_{r}\right),
\end{aligned}
$$

thus $0 \leq n_{B 1} \leq \min \left(E h, C_{r}\right) /\left(C_{s}-r_{s}\right)$.

2.3. An Illustrating Numerical Example. One numerical example is used to illustrate the application of Algorithm A for deriving the Bayesian sampling plan.

Example 1. A batch of $N=10,000$ items is presented for acceptance sampling. It is assumed that $(\alpha, \beta)=(2,1), C_{s}=$ 1.2, $r_{s}=0.2, C_{r}=6, C_{t}=1$, and $h(\lambda)=\lambda^{2}$. The goal is to find the Bayesian sampling plan for the acceptance sampling. Thus, $E h \equiv \int h(\lambda) g(\lambda) d \lambda=\alpha(\alpha+1) / \beta^{2}=6, \min \left(E h, C_{r}\right)=$ 6 , and $N^{*}=\left\langle\min \left(C_{r}, E h\right) /\left(C_{s}-r_{s}\right)\right\rangle=\langle 6 /(1.2-0.2)\rangle=5$. So, we only need to consider cases where $1 \leq n \leq N^{*}=5$. Since $a_{0}=a_{1}=0, a_{2}=1$ and $(\alpha, \beta)=(2,1)$, for each $n$,

$$
\begin{array}{r}
c(d)=\left(\sqrt{\frac{(d+2)(d+3)(1-n / N)}{C_{r}}}-1\right) \vee 0, \\
0 \leq d \leq n .
\end{array}
$$

A straightforward computation shows

$$
\begin{aligned}
S^{1,2}( & \left.\delta_{B 1} \mid n, n,(n \times 0), \infty\right) \\
= & \frac{(1-n / N) \beta^{\alpha}}{\Gamma(\alpha)} \sum_{j=0}^{n-1} \frac{(c(n))^{j} \Gamma(j+\alpha+2)}{j !(c(n)+\beta)^{j+\alpha+2}} \\
& +C_{r}\left\{1-\frac{\beta^{\alpha}}{\Gamma(\alpha)} \sum_{j=0}^{n-1} \frac{(c(n))^{j} \Gamma(j+\alpha)}{j !(c(n)+\beta)^{j+\alpha}}\right\} \\
= & \left(1-\frac{n}{N}\right) \sum_{j=0}^{n-1} \frac{(c(n))^{j} \Gamma(j+4)}{j !(c(n)+1)^{j+4}} \\
& +C_{r}\left(1-\sum_{j=0}^{n-1} \frac{(c(n))^{j} \Gamma(j+2)}{j !(c(n)+1)^{j+2}}\right) .
\end{aligned}
$$

Note that, $c(0)=0$. Hence, $C_{t} E\left[\min \left(X_{(1): n}, c(0) / n\right)\right]=0$.
As $n=1, c(1)=\sqrt{2 \times 0.9999}-1, S^{1,2}\left(\delta_{B 1} \mid 1,1,(0), \infty\right)=$ 4.49985. Thus,

$$
\begin{aligned}
S^{1,2} & \left(\delta_{B 1} \mid 1,1,(0), \infty\right)+n\left(C_{s}-r_{s}\right) \\
& =4.49985+1=5.49985<\min \left(C_{r}, E h\right)=6 .
\end{aligned}
$$

As $n=2, c(2)=\sqrt{9.998 / 3}-1, S^{1,2}\left(\delta_{B 1} \mid 2,2,(2 \times 0), \infty\right)=$ 4.08842. So,

$$
\begin{aligned}
& S^{1,2}\left(\delta_{B 1} \mid 2,2,(2 \times 0), \infty\right)+n\left(C_{s}-r_{s}\right) 4.08842+2 \\
& \quad=6.08842>\min \left(C_{r}, E h\right)=6 .
\end{aligned}
$$

As $n=3, c(3)=\sqrt{5 \times 0.9997}-1, S^{1,2}\left(\delta_{B 1} \mid 3,3,(3 \times\right.$ $0), \infty)=3.88991$ and

$$
\begin{aligned}
S^{1,2} & \left(\delta_{B 1} \mid 3,3,(3 \times 0), \infty\right)+n\left(C_{s}-r_{s}\right) \\
& =3.88991+3=6.888991>\min \left(C_{r}, E h\right)=6 .
\end{aligned}
$$

Similarly, for $n=4$ and 5, straightforward computations yield that

$$
\begin{aligned}
S^{1,2}\left(\delta_{B 1} \mid n, n,(n \times 0), \infty\right)+n\left(C_{s}-r_{s}\right) & >\min \left(C_{r}, E h\right) \\
& =6 .
\end{aligned}
$$

Hence, for finding the Bayesian sampling plan, by Step 2 of Algorithm A, it suffices to consider the type of sampling plans $\left(1,1,(0), T, \delta_{B 1}\right)$.

For the sampling plan $\left(1,1,(0), T, \delta_{B 1}\right), Y_{1}(1,1, d, T)=$ $X I(X \leq T)+T I(X>T)$, where $X$ is an exponential random variable having mean $\theta=1 / \lambda$. For $T>c(1)$, we have $S^{1,2}\left(\delta_{B 1} \mid 1,1,(0), T\right)=S^{1,2}\left(\delta_{B 1} \mid 1,1,(0), c(1)\right)$. Since both $E[D \mid n, T]$ and $E\left[\min \left(Y_{1: 1,1}, T\right)\right]$ are increasing in $T$, we have $R\left(1,1,(0), T, \delta_{B 1}\right) \geq R\left(1,1,(0), c(1), \delta_{B 1}\right)$ for all $T>c(1)$. So, for searching the best Bayesian sampling plan, it suffices to consider those sampling plans $\left(1,1,(0), T, \delta_{B 1}\right)$ where $0=$ $c(0) \leq T \leq c(1)$. Now, for the sampling plan $\left(1,1,(0), T, \delta_{B 1}\right)$,

$$
\begin{aligned}
& E[D \mid n, T]=\iint_{0}^{T} \lambda e^{-\lambda x} d x g(\lambda) d \lambda=1-\frac{\beta^{\alpha}}{(T+\beta)^{\alpha}}, \\
& E\left[\min \left(Y_{1: 1,1}, T\right)\right] \\
& \quad=\int\left[\int_{0}^{T} y \lambda e^{-\lambda y} d y+T \int_{T}^{\infty} \lambda e^{-\lambda y} d y\right] g(\lambda) d \lambda \\
& =\frac{\beta}{\alpha-1}-\frac{\beta^{\alpha}}{(\alpha-1)(T+\beta)^{\alpha-1}},
\end{aligned}
$$




$$
\begin{aligned}
S_{1} & =E\left[C_{r}\left(1-\delta_{B}(D, \underline{Y}(D))\right)\right]=C_{r} \iint_{0}^{T} \lambda e^{-\lambda x} d x g(\lambda) d \lambda \\
& =C_{r}\left(1-\frac{\beta^{\alpha}}{(T+\beta)^{\alpha}}\right), \\
S_{2} & =E\left[\delta_{B}(D, \underline{Y}(D))\left(1-\frac{1}{10.000}\right) h(\Lambda)\right] \\
& =0.9999 \int h(\lambda) \int_{T}^{\infty} \lambda e^{-\lambda x} d x g(\lambda) d \lambda \\
& =\frac{0.9999 \alpha(\alpha+1) \beta^{\alpha}}{(T+\beta)^{\alpha+2}} .
\end{aligned}
$$

Therefore,

$$
\begin{aligned}
R & \left(1,1,(0), T, \delta_{B}\right) \\
= & S_{1}+S_{2}+n\left(C_{s}-r_{s}\right)+r_{s} E[D]+C_{t} E\left[\min \left(Y_{1: 1,1}, T\right)\right] \\
= & C_{r}\left(1-\frac{\beta^{\alpha}}{(T+\beta)^{\alpha}}\right)+0.9999 \times \frac{\alpha(\alpha+1) \beta^{\alpha}}{(T+\beta)^{\alpha+2}}+1 \\
& +\left(1-\frac{\beta^{\alpha}}{(T+\beta)^{\alpha}}\right) r_{s}+\left(\frac{\beta}{\alpha-1}-\frac{\beta^{\alpha}}{(\alpha-1)(T+\beta)^{\alpha-1}}\right) C_{t} .
\end{aligned}
$$

Numerical computation is used for finding the $T_{B 1}$ to minimize $R\left(1,1,(0), T, \delta_{B 1}\right)$ of (35) among all $T$ such that $0=$ $c(0) \leq T \leq c(1)$. It is found that $T_{B 1}=0.3831$ and $\left(1,1,(0), T_{B 1}, \delta_{B 1}\right)$ is the Bayesian sampling plan. With this $T_{B 1}$ value, a computation shows $S_{1}=2.86351, S_{2}=1.63943$, $S_{3}=1, S_{4}=0.09545, S_{5}=0.2770$, and $R\left(1,1,(0), T_{B 1}, \delta_{B 1}\right)=$ 5.87539 .

\section{Derivation of the Bayesian Sampling Plan $\mathrm{BSP}_{2}$}

For the type-II APHC, during the experiment, for each $j=$ $1, \ldots, m$, when the $j$ th failure $Y_{j: m, n}$ is observed, if $Y_{j: m, n} \leq T$, immediately after the failure, $R_{j}$ functioning items are randomly removed from the life test; if $Y_{j: m, n}>T$ and $j<$ $m$, do not follow the prespecified censoring scheme to remove $R_{j}$ functioning items from the life test; instead, continue to observe failures without any further withdrawals; and if $Y_{m: m, n}>T$, immediately remove all the remaining functioning items and terminate the experiment. Let $K$ denote the number of failures before time $T$. Thus, $0 \leq K \leq m$. The duration of the life test experiment is $\tau=Y_{m: m, n}$. The observable variables $(K, \underline{Y}(m))=\left(K, Y_{1: m, n}, \ldots, Y_{m: m, n}\right)$ have a joint probability density function $f_{2}(k, \underline{y}(m) \mid \lambda)$ given as follows:

$$
f_{2}(k, \underline{y}(m) \mid \lambda)=A_{2}(n, m, k) \lambda^{m} e^{-\lambda y_{2}(n, m, k, T)},
$$

where $y_{2}(n, m, k, T)$ is an observed value of $Y_{2}(n, m, K, T)$, where

$$
\begin{aligned}
Y_{2}(n, m, K, T) & \left\{\begin{array}{cl}
Y_{1: m, n}+\cdots+Y_{m-1: m, n} \\
\quad+(n-m-1) Y_{m: m, n}, & \text { if } K=0 ; \\
\sum_{i=1}^{K}\left(R_{i}+1\right) Y_{i}+\sum_{i=K+1}^{m} Y_{i: m, n} & \\
+\left(n-m-n_{K}\right) Y_{m: m, n}, & \text { if } 1 \leq K \leq m-1 ; \\
\sum_{i=1}^{m}\left(R_{i}+1\right) Y_{i: m, n}, & \text { if } K=m
\end{array}\right.
\end{aligned}
$$

and $A_{2}(n, m, k)=\prod_{i=1}^{m}\left(n-i+1-\sum_{l=1}^{\max (i-1, k)} R_{l}\right)$.

3.1. A Bayes Decision Function $\delta_{B 2}$. Let

$$
\begin{gathered}
f_{2}(k, \underline{y}(m) \mid \lambda)=\int f_{2}(k, \underline{y}(m) \mid \lambda) g(\lambda) d \lambda, \\
g_{2}(\lambda \mid k, \underline{y}(m))=\frac{f_{2}(k, \underline{y}(m) \mid \lambda) g(\lambda)}{f_{2}(k, \underline{y}(m))}, \\
\varphi_{2}(k, \underline{y}(m))=\left(1-\frac{n}{N}\right) \int h(\lambda) g_{2}(\lambda \mid k, \underline{y}(m)) d \lambda .
\end{gathered}
$$

For each observed variable $(k, \underline{y}(m))$, define

$$
\delta_{B 2}(k, \underline{y}(m))= \begin{cases}1 & \text { if } \varphi_{2}(k, \underline{y}(m))<C_{r} ; \\ 0, & \text { otherwise. }\end{cases}
$$

Note that

$$
\begin{aligned}
S^{1,2} & \left(\delta \mid n, m,\left(R_{1}, \ldots, R_{m}\right), T\right) \\
& =S_{1}+S_{2}=E\left[\delta(D, \underline{Y}(D))\left(h(\Lambda)-C_{r}\right)\right]+C_{r} \\
& \equiv S\left(\delta \mid n, m,\left(R_{1}, \ldots, R_{m}\right), T\right)+C_{r} .
\end{aligned}
$$

Similar to Theorem 1, we can obtain the following theorem.

Theorem 3. $\delta_{B 2}$ is a Bayes decision function in the sense that, for each fixed sampling parameters $\left(n, m,\left(R_{1}, \ldots, R_{m}\right), T\right)$, $S\left(\delta_{B 2} \mid n, m,\left(R_{1}, \ldots, R_{m}\right), T\right) \leq S\left(\delta \mid n, m,\left(R_{1}, \ldots, R_{m}\right), T\right)$ for all decision functions $\delta$.

3.2. Derivation of Bayesian Sampling Plans. From (2), we have

$$
\begin{aligned}
R(n, m, & \left.\left(R_{1}, \ldots, R_{m}\right), T, \delta_{B 2}\right)=S_{1}+S_{2}+S_{3}+S_{4}+S_{5}, \\
S_{1} & =E\left[C_{r}\left(1-\delta_{B 2}(K, \underline{Y}(m))\right)\right], \\
S_{2} & =E\left[\delta_{B 2}(K, \underline{Y}(m))\left(1-\frac{n}{N}\right) h(\Lambda)\right] \\
S_{3} & =n\left(C_{s}-r_{s}\right), \\
S_{4} & =r_{s} m, \\
S_{5} & =C_{t} E\left[Y_{m: m, n}\right] .
\end{aligned}
$$


Note that $Y_{m: m, n} \geq X_{(m): n}$, where $X_{(m): n}$ is the $m$ th ordered statistic of the lifetime variables of the $n$ items put on life test. Similar to the inequality of (19), the following inequality holds:

$$
\begin{aligned}
R\left(n, m,\left(R_{1}, \ldots, R_{m}\right), T, \delta_{B 2}\right) \\
\geq S^{1,2}\left(\delta_{B 2} \mid n, n,(n \times 0), 0\right)+n\left(C_{s}-r_{s}\right)+r_{s} m \\
\quad+C_{t} E\left[X_{(m): n}\right] .
\end{aligned}
$$

So if

$$
\begin{aligned}
& S^{1,2}\left(\delta_{B 2} \mid n, n,(n \times 0), 0\right)+n\left(C_{s}-r_{s}\right)+r_{s} m \\
& +C_{t} E\left[X_{(m): n}\right] \geq \min \left(E h, C_{r}\right),
\end{aligned}
$$

then $\left(n, m,\left(R_{1}, \ldots, R_{m}\right), T, \delta_{B 2}\right)$ is not a Bayesian sampling plan. With this property, we propose Algorithm $B$ for deriving a Bayesian sampling plan as follows.

Algorithm B. Define $N^{*}=\left\langle\min \left(C_{r}, E h\right) /\left(C_{s}-r_{s}\right)\right\rangle$, where $\langle x\rangle$ denotes the largest integer less than $x$.

Step 1. For each $n$ and $m$ satisfying $1 \leq m \leq n \leq N^{*}$ and

$$
\begin{aligned}
& S^{1,2}\left(\delta_{B 2} \mid n, n,(n \times 0), 0\right)+n\left(C_{s}-r_{s}\right)+r_{s} m \\
& \quad+C_{t} E\left[X_{(m): n}\right]<\min \left(E h, C_{r}\right),
\end{aligned}
$$

construct all type-II progressive hybrid censoring schemes $\left(n, m,\left(R_{1}, \ldots, R_{m}\right), T\right)$.

Go to Step 2.

If there is no pairs of $(n, m)$ satisfying the restrictions of $(45)$, take $n_{B 2}=0$. Then, adopt the sampling plan $\left(0,0,(0), 0, \delta_{B 2}\right)$ with no sample data. Go to Step 8.

Step 2. For each fixed $\left(n, m,\left(R_{1}, \ldots, R_{m}\right), T\right)$, derive the Bayes decision function $\delta_{B 2} \equiv \delta_{B 2}\left(n, m,\left(R_{1}, \ldots, R_{m}\right), T\right)$. Note that if $R\left(n, m,\left(R_{1}, \ldots, R_{m}\right), T, \delta_{B 2}\right)>\min \left(E h, C_{r}\right)$, then $\left(n, m,\left(R_{1}, \ldots, R_{m}\right), T, \delta_{B 2}\right)$ is not a Bayesian sampling plan.

If $R\left(n, m,\left(R_{1}, \ldots, R_{m}\right), T, \delta_{B 2}\right)>\min \left(E h, C_{r}\right)$ for all $\left(n, m,\left(R_{1}, \ldots, R_{m}\right), T, \delta_{B 2}\right)$, take $n_{B 2}=0$ and adopt the sampling plan $\left(0,0,(0), 0, \delta_{B 2}\right)$. Go to Step 8 .

Otherwise, go to Step 3.

Step 3. For each fixed $\left(n, m,\left(R_{1}, \ldots, R_{m}\right)\right)$, find the time $T_{B 2} \geq$ 0 such that

$$
\begin{aligned}
R & \left(n, m,\left(R_{1}, \ldots, R_{m}\right), T_{B 2}, \delta_{B 2}\right) \\
\quad & \inf _{T \geq 0}\left\{R\left(n, m,\left(R_{1}, \ldots, R_{m}\right), T, \delta_{B 2}\right)\right\} .
\end{aligned}
$$

Step 4. For each fixed $(n, m)$, find the censoring scheme $\left(R_{1}^{*}, \ldots, R_{m}^{*}\right)$ such that

$$
\begin{aligned}
R & \left(n, m,\left(R_{1}^{*}, \ldots, R_{m}^{*}\right), T_{B 2}, \delta_{B 2}\right) \\
\quad & =\inf _{\left(R_{1}, \ldots, R_{m}\right)}\left\{R\left(n, m,\left(R_{1}, \ldots, R_{m}\right), T_{B 2}, \delta_{B 2}\right)\right\} .
\end{aligned}
$$

Step 5. For each $n$, find the $m_{B 2}$ such that

$$
\begin{aligned}
R & \left(n, m_{B 2},\left(R_{1}^{*}, \ldots, R_{m_{B 2}}^{*}\right), T_{B 2}, \delta_{B 2}\right) \\
& =\min _{1 \leq m \leq n} R\left(n, m,\left(R_{1}^{*}, \ldots, R_{m}^{*}\right), T_{B 2}, \delta_{B 2}\right) .
\end{aligned}
$$

Step 6. Find $n_{B 2}, 0 \leq n_{B 2} \leq N^{*}$, such that

$$
\begin{aligned}
R & \left(n_{B 2}, m_{B 2},\left(R_{1}^{*}, \ldots, R_{m_{B 2}}^{*}\right), T_{B 2}, \delta_{B 2}\right) \\
& =\min _{1 \leq n \leq N^{*}} R\left(n, m_{B 2},\left(R_{1}^{*}, \ldots, R_{m_{B 2}}^{*}\right), T_{B 2}, \delta_{B 2}\right) .
\end{aligned}
$$

Step 7. If $R\left(n_{B 2}, m_{B 2},\left(R_{1}^{*}, \ldots, R_{m_{B 2}}^{*}\right), T_{B 2}, \delta_{B 2}\right)<R(0,0,(0), 0$, $\left.\delta_{B 2}\right)$, then propose the sampling plan $\left(n_{B 2}, m_{B 2},\left(R_{1}^{*}, \ldots\right.\right.$, $\left.\left.R_{m_{B 2}}^{*}\right), T_{B 2}, \delta_{B 2}\right)$; otherwise, $\left(0,0,(0), 0, \delta_{B 2}\right)$ is the proposed sampling plan.

Step 8. When $n_{B 2}=0,\left(0,0,(0), 0, \delta_{B 2}\right)$ is the proposed sampling plan.

Analogous to Theorem 2, the following theorem holds.

Theorem 4. $\left(n_{B 2}, m_{B 2},\left(R_{1}^{*}, \ldots, R_{m_{B 2}}^{*}\right), T_{B 2}, \delta_{B 2}\right)$ is an optimal sampling plan in the sense that $R\left(n_{B 2}, m_{B 2},\left(R_{1}^{*}, \ldots, R_{m_{B 2}}^{*}\right)\right.$, $\left.T_{B 2}, \delta_{B 2}\right) \leq R\left(n, m,\left(R_{1}, \ldots, R_{m}\right), T, \delta\right)$ for all sampling plans $\left(n, m,\left(R_{1}, \ldots, R_{m}\right), T, \delta\right)$.

3.3. An Illustrating Example. Before presenting an example, we will provide some useful results.

(a) For any sampling plan $\left(n, m,\left(R_{1}, \ldots, R_{m}\right), T, \delta_{B 2}\right)$, the following inequality holds:

$$
\begin{array}{r}
S^{1,2}\left(\delta_{B 2} \mid n, m,\left(R_{1}, \ldots, R_{m}\right), T\right) \\
\geq S^{1,2}\left(\delta_{B 2} \mid n, n,(n \times 0), 0\right) ;
\end{array}
$$

(b) for any sampling plan $\left(n, m,\left(R_{1}, \ldots, R_{m}\right), T, \delta\right)$, $E\left[X_{(m): n}\right] \leq E\left[Y_{m: m, n}\right] ;$

(c) for any sampling plan $\left(n, m,\left(R_{1}, \ldots, R_{m}\right), T, \delta_{B 2}\right)$,

$$
\begin{aligned}
R\left(n, m,\left(R_{1}, \ldots, R_{m}\right), T, \delta_{B 2}\right) \\
\geq S^{1,2}\left(\delta_{B 2} \mid n, n,(n \times 0), 0\right)+n\left(C_{s}-r_{s}\right)+m r_{s} \\
\quad+C_{t} E\left[X_{(m): n}\right] ;
\end{aligned}
$$

(d) one has $R\left(n, n,(n \times 0), 0, \delta_{B 2}\right)=S^{1,2}\left(\delta_{B 2} \mid n, n,(n \times\right.$ $0), 0)+n\left(C_{s}-r_{s}\right)+n r_{s}+E\left[X_{(n): n}\right]$

(e) one has $E\left[X_{(m): n}\right]=(n ! /(m-1) !(n-m) !)(\beta /(\alpha-$ 1)) $\sum_{k=0}^{m-1}\left(\begin{array}{c}m-1 \\ k\end{array}\right)\left((-1)^{k} /(n-m+1+k)^{2}\right)$.

Example 2. The model studied in Example 1 is applied here to illustrate the application of Algorithm B for searching a Bayesian sampling plan. From Example 1, we have that $(\alpha, \beta)=(2,1), C_{s}=1.2, r_{s}=0.2, C_{r}=6,2, C_{t}=0.1$, and $h(\lambda)=\lambda^{2}$. The goal is to find the Bayesian sampling plan for the acceptance sampling. Thus, $E h \equiv \int h(\lambda) g(\lambda) d \lambda=\alpha(\alpha+$ 
1) $/ \beta^{2}=6, \min \left(E h, C_{r}\right)=6$, and $N^{*}=\left\langle\min \left(C_{r}, E h\right) /\left(C_{s}-\right.\right.$ $\left.\left.r_{s}\right)\right\rangle=\langle 6 /(1.2-0.2)\rangle=5$. So, we only need to consider cases where $1 \leq n \leq N^{*}$. With the numerical values provided in Example 1, we see that, for each $(n, m)$ with $2 \leq m \leq n \leq 5$, the following inequality holds:

$$
\begin{aligned}
R\left(n, m,\left(R_{1}, \ldots, R_{m}\right), T, \delta_{B 2}\right) \\
\geq S^{1,2}\left(\delta_{B 2} \mid n, n,(n \times 0), \infty\right)+n\left(C_{s}-r_{s}\right)+m r_{s} \\
\quad+C_{t} E\left[X_{(n): n}\right] \geq \min \left(E h, C_{r}\right)=6 .
\end{aligned}
$$

Thus, for each $(n, m), 2 \leq m \leq n \leq 6,\left(n, m,\left(R_{1}, \ldots, R_{m}\right)\right.$, $\left.T, \delta_{B 2}\right)$ is not a Bayesian sampling plan. Therefore, for searching a Bayesian sampling plan, we only need to consider the sampling plan $\left(1,1,(0), 0, \delta_{B 2}\right)$. Since

$$
E\left[X_{(1): 1}\right]=\frac{\beta}{\alpha-1} \sum_{k=0}^{m-1}\left(\begin{array}{c}
m-1 \\
k
\end{array}\right) \frac{(-1)^{k}}{(n-m+1+k)^{2}}=1 \text {, }
$$

we have

$$
\begin{aligned}
R & \left(1,1,(0), 0, \delta_{B 2}\right) \\
& =S^{1,2}\left(\delta_{B 2} \mid 1,1,(0), 0\right)+\left(C_{s}-r_{s}\right)+r_{s}+0.1 \times E\left[X_{(1): 1}\right] \\
& =4.49985+1+0.2+0.1=5.79985<\min \left(E h, C_{r}\right)=6
\end{aligned}
$$

Therefore, $\left(1,1,(0), 0, \delta_{B 2}\right)$ is the Bayesian sampling plan for the concerned acceptance sampling.

\section{Comparison with Lin and Huang's [3] Sampling Plans}

4.1. Comparison with Type-I APHC. Lin and Huang [3] have studied the acceptance sampling with the loss $L$ and with type-I APHC. Lin and Huang [3] considered a type of decision function $\delta^{\mathrm{LH} 1}$, which is defined below. When $D \geq 1$, the MLE of the expected lifetime $\theta=1 / \lambda$ is $\widetilde{\theta}_{1}=Y(n, m$, $D, T) / D$. The decision function $\delta^{\mathrm{LH} 1}$ is defined as

$$
\begin{aligned}
& \text { When } D \geq 1, \delta^{\mathrm{LH} 1}(D, \underline{Y}(D))=1 \text {, } \\
& \text { if } \tilde{\theta}_{1} \geq \xi ; \text { and } 0 \text {, otherwise; }
\end{aligned}
$$

When $D=0$, no decision is made.

A sampling plan with the type of decision functions is denoted by $\left(n, m,\left(R_{1}, \ldots, R_{m}\right), T, \xi\right)$ (or by $\left(n, m,\left(R_{1}, \ldots\right.\right.$, $\left.\left.\left.R_{m}\right), T, \delta^{\mathrm{LH} 1}\right)\right)$. The Bayes risk of SP $=\left(n, m,\left(R_{1}, \ldots, R_{m}\right)\right.$, $T, \xi)$ can be presented as

$$
\begin{aligned}
& R\left(n, m,\left(R_{1}, \ldots, R_{m}\right), T, \xi\right) \\
& \quad=S_{1}(\mathrm{SP})+S_{2}(\mathrm{SP})+S_{3}(\mathrm{SP})+S_{4}(\mathrm{SP})+S_{5}(\mathrm{SP})
\end{aligned}
$$

where

$$
\begin{aligned}
& S_{1}(\mathrm{SP})=C_{r} E\left[1-\delta^{\mathrm{LH} 1}(D, \underline{Y}(D)) \mid D>0\right], \\
& S_{2}(\mathrm{SP})=\left(1-\frac{n}{N}\right) E\left[h(\lambda) \delta^{\mathrm{LH} 1}(D, \underline{Y}(D)) \mid D>0\right], \\
& S_{3}(\mathrm{SP})=n\left(C_{s}-r_{s}\right), \\
& S_{4}(\mathrm{SP})=r_{s} E[D] \\
& S_{5}(\mathrm{SP})=C_{t} T .
\end{aligned}
$$

Lin and Huang [3] claimed having derived the Bayesian sampling plans $\mathrm{SP}^{o}=\left(n^{o}, m^{o},\left(R_{1}^{o}, \ldots, R_{m^{o}}^{o}\right), T^{o}, \xi^{o}\right)$. The values of parameters of the sampling plans $\left(n^{o}, m^{o},\left(R_{1}^{o}\right.\right.$, $\left.\left.\ldots, R_{m^{o}}^{o}\right), T^{o}, \xi^{o}\right)$ and the associated Bayes risks have been provided in Table 4 of Lin and Huang [3] (also, see Table 1 of the present paper). We note that there are some minor errors regarding the values of MBR. Thus, to make an easy comparison, with the provided values of parameters of the sampling plans $\left(n^{o}, m^{o},\left(R_{1}^{o}, \ldots, R_{m^{o}}^{o}\right), T^{o}, \xi^{o}\right)$, the corresponding $S_{j}(\mathrm{SP})$ values, $j=1, \ldots, 5$, and the Bayes risks $R\left(\mathrm{SP}^{\circ}\right)$ are calculated and reported in Table 2 . We will present certain propositions to verify the correctness of the sampling plans $\mathrm{SP}^{o}$.

Proposition 5. Suppose that a sampling plan $S P^{o}=\left(n^{o}, m^{o}\right.$, $\left.\left(R_{1}^{o}, \ldots, R_{m^{o}}^{o}\right), T^{o}, \xi^{o}\right)$ is such that $R\left(S P^{o}\right)-S_{3}\left(S P^{o}\right)-\min ((1-$ $\left.\left.n^{o} / N\right) E h, C_{r}\right)>0$. Then, this sampling plan $S P^{o}$ is not a Bayesian sampling plan.

Proof. It suffices to find a sampling plan $\mathrm{SP}^{*}=\left(n^{o}, m^{o}\right.$, $\left.\left(R_{1}^{o}, \ldots, R_{m^{o}}^{o}\right), T^{*}, \xi^{*}\right)$ to dominate the $\mathrm{SP}^{o}$. First, note that both $S_{4}=r_{s} E\left[\min \left(m, D_{1}\right)\right]$ and $S_{5}=C_{t} E\left[\min \left(X_{m: m, n}, T\right)\right]$ are increasing functions of $T$ and tend to zero as $T$ approaches zero.

If $\left(1-n^{o} / N\right) E h \leq C_{r}$, we let $\xi^{*}=0$ and choose $0<T^{*}<$ $T^{o}$ to be small enough such that

$$
S_{4}\left(\mathrm{SP}^{*}\right)+S_{5}\left(\mathrm{SP}^{*}\right)<R\left(\mathrm{SP}^{o}\right)-S_{3}\left(\mathrm{SP}^{o}\right)-\left(1-\frac{n^{o}}{N}\right) E h .
$$

If $\left(1-n^{o} / N\right) E h>C_{r}$, we let $\xi^{*}=n^{o} \xi^{o}$ and choose $0<T^{*}<\xi^{o}$ to be small enough such that

$$
S_{4}\left(\mathrm{SP}^{*}\right)+S_{5}\left(\mathrm{SP}^{*}\right)<R\left(\mathrm{SP}^{o}\right)-S_{3}\left(\mathrm{SP}^{o}\right)-C_{r}
$$

Now, we will show that $\mathrm{SP}^{*}$ dominates $\mathrm{SP}^{o}$. Note that $S_{3}\left(\mathrm{SP}^{*}\right)=S_{3}\left(\mathrm{SP}^{o}\right)$. 
TABLE 1: Lin and Huang's [3] sampling plans and the associated MBR for type-I APHCS with $a_{0}=a_{1}=1, C_{s}=1, r_{s}=0.5$, and $C_{5}=1-n^{\circ} / 1000$.

\begin{tabular}{|c|c|c|c|c|c|c|c|c|c|c|c|}
\hline Case & $(\alpha$ & $\beta$ & $a_{2}$ & $C_{t}$ & $\left.C_{r}\right)$ & $\left(n^{o}\right.$ & $m^{o}$ & $\left(\underline{R}^{o}\right)$ & $T^{o}$ & $\left.\xi^{o}\right)$ & MBR \\
\hline 1 & 1.5 & 2.0 & 1.0 & 2 & 5 & 2 & 1 & (1) & 0.0340 & 0.0170 & 3.7787 \\
\hline 2 & 2.0 & 2.0 & 1.0 & 2 & 5 & 1 & 1 & $(0)$ & 0.0888 & 0.0127 & 4.4285 \\
\hline 3 & 2.5 & 2.0 & 1.0 & 2 & 5 & 1 & 1 & (0) & 0.0478 & 0.0102 & 5.1695 \\
\hline 4 & 3.0 & 2.0 & 1.0 & 2 & 5 & 1 & 1 & (0) & 0.0169 & 0.1713 & 5.5387 \\
\hline 5 & 3.5 & 2.0 & 1.0 & 2 & 5 & 1 & 1 & (0) & 0.0145 & 0.6335 & 5.5112 \\
\hline 6 & 4.0 & 2.0 & 1.0 & 2 & 5 & 1 & 1 & (0) & 0.0127 & 0.2589 & 5.5379 \\
\hline 7 & 5.0 & 2.0 & 1.0 & 2 & 5 & 1 & 1 & (0) & 0.0102 & 0.1489 & 5.5186 \\
\hline 8 & 1.5 & 0.8 & 1.0 & 2 & 5 & 1 & 1 & (0) & 0.0136 & 4.7503 & 5.5397 \\
\hline 9 & 1.5 & 1.0 & 1.0 & 2 & 5 & 1 & 1 & $(0)$ & 0.0170 & 0.4695 & 5.5463 \\
\hline 10 & 1.5 & 3.0 & 1.0 & 2 & 5 & 2 & 1 & (1) & 0.0511 & 0.0255 & 3.0253 \\
\hline 11 & 1.5 & 4.0 & 1.0 & 2 & 5 & 2 & 1 & (1) & 0.0681 & 0.0340 & 2.7856 \\
\hline 12 & 1.5 & 5.0 & 1.0 & 2 & 5 & 2 & 1 & (1) & 0.0851 & 0.0426 & 2.6627 \\
\hline 13 & 1.5 & 10.0 & 1.0 & 2 & 5 & 2 & 1 & (1) & 0.1702 & 0.0851 & 2.5743 \\
\hline 14 & 1.5 & 2.0 & 2.0 & 2 & 5 & 1 & 1 & (0) & 0.1008 & 0.0170 & 4.5823 \\
\hline 15 & 1.5 & 2.0 & 2.5 & 2 & 5 & 1 & 1 & $(0)$ & 0.0823 & 0.0170 & 4.9578 \\
\hline 16 & 1.5 & 2.0 & 3.0 & 2 & 5 & 1 & 1 & (0) & 0.0582 & 0.0170 & 5.3073 \\
\hline 17 & 1.5 & 2.0 & 5.0 & 2 & 5 & 1 & 1 & (0) & 0.0340 & 6.1106 & 5.5806 \\
\hline 18 & 1.5 & 2.0 & 7.5 & 2 & 5 & 1 & 1 & $(0)$ & 0.0340 & 6.3531 & 5.5806 \\
\hline 19 & 1.5 & 2.0 & 10.0 & 2 & 5 & 1 & 1 & (0) & 0.0340 & 6.1438 & 5.5806 \\
\hline 20 & 1.5 & 2.0 & 1.0 & 0 & 5 & 1 & 1 & (0) & 0.4109 & 0.0170 & 3.4057 \\
\hline 21 & 1.5 & 2.0 & 1.0 & 1 & 5 & 1 & 1 & (0) & 0.1738 & 0.0170 & 3.6471 \\
\hline 22 & 1.5 & 2.0 & 1.0 & 3 & 5 & 2 & 1 & (1) & 0.0340 & 0.0170 & 3.8128 \\
\hline 23 & 1.5 & 2.0 & 1.0 & 4 & 5 & 2 & 1 & (1) & 0.0340 & 0.0170 & 3.8468 \\
\hline 24 & 1.5 & 2.0 & 1.0 & 5 & 5 & 2 & 1 & (1) & 0.0340 & 0.0170 & 3.8808 \\
\hline 25 & 1.5 & 2.0 & 1.0 & 10 & 5 & 2 & 1 & (1) & 0.0340 & 0.0170 & 4.0511 \\
\hline 26 & 1.5 & 2.0 & 1.0 & 2 & 10 & 2 & 2 & $(2 * 0)$ & 0.0340 & 0.0170 & 3.8111 \\
\hline 27 & 1.5 & 2.0 & 1.0 & 2 & 15 & 2 & 1 & (1) & 0.0340 & 0.0170 & 3.8434 \\
\hline 28 & 1.5 & 2.0 & 1.0 & 2 & 20 & 2 & 1 & (1) & 0.0348 & 0.0170 & 3.8757 \\
\hline 29 & 1.5 & 2.0 & 1.0 & 2 & 30 & 2 & 1 & (1) & 0.0447 & 0.0170 & 3.9316 \\
\hline 30 & 1.5 & 2.0 & 1.0 & 2 & 50 & 2 & 1 & (1) & 0.0597 & 0.0170 & 4.0179 \\
\hline 31 & 1.5 & 2.0 & 1.0 & 2 & 100 & 2 & 1 & (1) & 0.0867 & 0.0170 & 4.1758 \\
\hline
\end{tabular}

MBR: the Bayes risk reported in Lin and Huang [3]. $\left(\underline{R}^{o}\right)=\left(R_{1}^{o}, \ldots, R_{m}^{o}\right)$.

For $\left(1-n^{o} / N\right) E h \leq C_{r}$ case, since $\xi^{*}=0$, we always accept the lot. So, we have $S_{1}\left(\mathrm{SP}^{*}\right)+S_{2}\left(\mathrm{SP}^{*}\right)=\left(1-n^{o} / N\right) E h$. Therefore,

$$
\begin{aligned}
R\left(\mathrm{SP}^{o}\right)-R\left(\mathrm{SP}^{*}\right) \\
=\left[R\left(\mathrm{SP}^{o}\right)-S_{3}\left(\mathrm{SP}^{o}\right)\right]-\left[R\left(\mathrm{SP}^{*}\right)-S_{3}\left(\mathrm{SP}^{*}\right)\right] \\
=\left[R\left(\mathrm{SP}^{o}\right)-S_{3}\left(\mathrm{SP}^{o}\right)\right] \\
-\left[S_{1}\left(\mathrm{SP}^{*}\right)+S_{2}\left(\mathrm{SP}^{*}\right)+S_{4}\left(\mathrm{SP}^{*}\right)+S_{5}\left(\mathrm{SP}^{*}\right)\right] \\
=\left[R\left(\mathrm{SP}^{o}\right)-S_{3}\left(\mathrm{SP}^{o}\right)\right] \\
-\left[S_{4}\left(\mathrm{SP}^{*}\right)+S_{5}\left(\mathrm{SP}^{*}\right)+\left(1-\frac{n^{o}}{N}\right) E h\right]>0 .
\end{aligned}
$$

Note that the last inequality is obtained due to the choice of the value of $T^{*}$ and (58).
Similarly, for $\left(1-n^{o} / N\right) E h>C_{r}$ case, we can also obtain that $R\left(\mathrm{SP}^{o}\right)-R\left(\mathrm{SP}^{*}\right)>0$.

Combining the preceding consequences leads to the conclusion that the sampling plan $\mathrm{SP}^{o}$ is not a Bayesian sampling plan.

After carefully examining the numerical values given in Table 2 , it is found that the inequality $R\left(\mathrm{SP}^{o}\right)-S_{3}\left(\mathrm{SP}^{o}\right)-$ $\min \left(\left(1-n^{o} / N\right) E h, C_{r}\right)>0$ holds for all cases studied in Table 1 . Thus, we conclude all the sampling plans given in Table 1 are non-Bayesian.

Proposition 6. Suppose that a sampling plan $S P^{o}=\left(n^{o}, m^{o}\right.$, $\left.\left(R_{1}^{o}, \ldots, R_{m^{o}}^{o}\right), T^{o}, \xi^{o}\right)$ is such that $n^{o} T^{o}<\xi^{o}$. Then, this sampling plan $S P^{o}$ is not a Bayesian sampling plan.

Proof. It suffices to find a sampling plan $\mathrm{SP}^{*}=$ $\left(n^{o}, m^{o},\left(R_{1}^{o}, \ldots, R_{m^{o}}^{o}\right), T^{*}, \xi^{*}\right)$ to dominate the $\mathrm{SP}^{o}$. Since $n^{o} T^{o}<\xi^{o}$, the sampling plan $\mathrm{SP}^{o}$ always rejects the lot. Thus, 
TABLE 2: Comparison of performance of $\left(n_{B 1}, m_{B 1},\left(R_{B 1}, \ldots, R_{B m_{B 1}}\right), T_{B 1}, \delta_{B 1}\right)$ and $\left(n^{o}, m^{o},\left(R_{1}^{o}, \ldots, R_{m^{o}}^{o}\right), T^{o}, \xi^{o}\right)$ for type-I APHCS sampling plans with $a_{0}=a_{1}=1, C_{s}=1, r_{s}=0.5$, and $C_{5}=1-n^{\circ} / 1000$.

\begin{tabular}{|c|c|c|c|c|c|c|c|c|}
\hline Case & $S_{1}$ & $S_{2}$ & $S_{3}$ & $S_{4}$ & $S_{5}$ & $R\left(\mathrm{SP}^{o}\right)$ & MBR & $R\left(\delta_{B}\right)$ \\
\hline 1 & 0.03232 & 2.65307 & 1.0 & 0.024969 & 0.0680 & 3.77836 & 3.7787 & 2.6875 \\
\hline 2 & 0.74270 & 2.96585 & 0.5 & 0.041609 & 0.1776 & 4.42776 & 4.4285 & 3.5000 \\
\hline 3 & 1.09222 & 3.45321 & 0.5 & 0.028669 & 0.0956 & 5.16970 & 5.1695 & 4.4375 \\
\hline 4 & 5.00000 & 0.00000 & 0.5 & 0.012464 & 0.0338 & 5.54626 & 5.5387 & 5.0000 \\
\hline 5 & 5.00000 & 0.00000 & 0.5 & 0.012483 & 0.0290 & 5.54148 & 5.5112 & 5.0000 \\
\hline 6 & 5.00000 & 0.00000 & 0.5 & 0.012501 & 0.0254 & 5.53790 & 5.5379 & 5.0000 \\
\hline 7 & 5.00000 & 0.00000 & 0.5 & 0.012557 & 0.0204 & 5.53296 & 5.5186 & 5.0000 \\
\hline 8 & 5.00000 & 0.00000 & 0.5 & 0.012484 & 0.0272 & 5.53968 & 5.5397 & 5.0000 \\
\hline 9 & 5.00000 & 0.00000 & 0.5 & 0.012484 & 0.0340 & 5.54648 & 5.5463 & 5.0000 \\
\hline 10 & 0.03226 & 1.89424 & 1.0 & 0.025017 & 0.1022 & 3.05372 & 3.0255 & 1.9167 \\
\hline 11 & 0.03227 & 1.59145 & 1.0 & 0.025005 & 0.1362 & 2.78493 & 2.7856 & 1.6094 \\
\hline 12 & 0.03243 & 1.43416 & 1.0 & 0.024997 & 0.1702 & 2.66179 & 2.6627 & 1.4500 \\
\hline 13 & 0.03236 & 1.17387 & 1.0 & 0.024997 & 0.3404 & 2.57163 & 2.5743 & 1.1875 \\
\hline 14 & 0.86992 & 2.97459 & 0.5 & 0.035551 & 0.2016 & 4.58166 & 4.5328 & 3.6250 \\
\hline 15 & 1.05811 & 3.20508 & 0.5 & 0.029348 & 0.1646 & 4.95713 & 4.9578 & 4.0938 \\
\hline 16 & 1.48282 & 3.18637 & 0.5 & 0.021057 & 0.1164 & 5.30664 & 5.3073 & 4.5625 \\
\hline 17 & 5.00000 & 0.00000 & 0.5 & 0.012484 & 0.0680 & 5.58048 & 5.5806 & 5.0000 \\
\hline 18 & 5.00000 & 0.00000 & 0.5 & 0.012484 & 0.0680 & 5.58048 & 5.5806 & 5.0000 \\
\hline 19 & 5.00000 & 0.00000 & 0.5 & 0.012484 & 0.0680 & 5.58048 & 5.5806 & 5.0000 \\
\hline 20 & 0.23970 & 2.54335 & 0.5 & 0.122214 & 0.0000 & 3.40526 & 3.4057 & 2.6875 \\
\hline 21 & 0.51853 & 2.39541 & 0.5 & 0.058749 & 0.1738 & 3.64649 & 3.6471 & 2.6875 \\
\hline 22 & 0.03232 & 2.65307 & 1.0 & 0.024969 & 0.1020 & 3.81236 & 3.8128 & 2.6875 \\
\hline 23 & 0.03232 & 2.65307 & 1.0 & 0.024969 & 0.1360 & 3.84636 & 3.8468 & 2.6875 \\
\hline 24 & 0.03230 & 2.65307 & 1.0 & 0.024969 & 0.1700 & 3.88033 & 3.8808 & 2.6875 \\
\hline 25 & 0.03232 & 2.65307 & 1.0 & 0.024969 & 0.3400 & 4.05036 & 4.0511 & 2.6875 \\
\hline 26 & 0.06464 & 2.65307 & 1.0 & 0.024969 & 0.0680 & 3.81068 & 3.8111 & 2.6875 \\
\hline 27 & 0.09696 & 2.65307 & 1.0 & 0.024969 & 0.0680 & 3.84300 & 3.8434 & 2.6875 \\
\hline 28 & 0.12643 & 2.65369 & 1.0 & 0.025544 & 0.0696 & 3.87526 & 3.8757 & 2.6875 \\
\hline 29 & 0.14945 & 2.65953 & 1.0 & 0.032612 & 0.0894 & 3.93100 & 3.9316 & 2.6875 \\
\hline 30 & 0.18998 & 2.61165 & 1.0 & 0.043161 & 0.1194 & 3.96419 & 4.0179 & 2.6875 \\
\hline 31 & 0.27042 & 2.61788 & 1.0 & 0.061671 & 0.1734 & 4.12337 & 4.1758 & 2.6875 \\
\hline
\end{tabular}

$R\left(\delta_{B}\right)$ : the Bayes risk of the sampling plan $\left(n_{B}, m_{B},\left(R_{B 1}, \ldots, R_{B m_{B}}\right), T_{B}, \delta_{B}\right)$.

$R\left(\mathrm{SP}^{o}\right)$ : the recalculated Bayes risk $R\left(n^{o}, m^{o},\left(R_{1}^{o}, \ldots, R_{m^{o}}^{o}\right), T^{o}, \xi^{o}\right)$.

MBR: the Bayes risk reported in Lin and Huang [3].

$S_{1}\left(\mathrm{SP}^{o}\right)=C_{r}$ and $S_{2}\left(\mathrm{SP}^{o}\right)=0$. Now, for the $\mathrm{SP}^{*}$, choose $0<$ $T^{*}<T^{o}$ and $\xi^{*}=\xi^{o}$. Since $0<T^{*}<T^{o}$, we have $S_{1}\left(\mathrm{SP}^{*}\right)=$ $S_{1}\left(\mathrm{SP}^{o}\right)=C_{r}, S_{2}\left(\mathrm{SP}^{*}\right)=S_{2}\left(\mathrm{SP}^{o}\right)=0, S_{3}\left(\mathrm{SP}^{*}\right)=S_{3}\left(\mathrm{SP}^{o}\right)$, $S_{4}\left(\mathrm{SP}^{*}\right)<S_{4}\left(\mathrm{SP}^{o}\right)$, and $S_{5}\left(\mathrm{SP}^{*}\right)<S_{5}\left(\mathrm{SP}^{o}\right)$. Therefore, we have $R\left(\mathrm{SP}^{*}\right)<R\left(\mathrm{SP}^{o}\right)$. Hence, $\mathrm{SP}^{o}$ is not a Bayesian sampling plan.

In Table 1, there are nine cases at each of which the inequality holds: $n^{o} T^{o}<\xi^{o}$. So, for those cases, the reported $\mathrm{SP}^{o}$ are not Bayesian sampling plans.

By applying Algorithm A, for each case studied in Table 1, we have derived the Bayesian sampling plan. It happens that, for all cases studied, the Bayesian sampling plans are $\left(0,0,(0), 0, \delta_{B 1}\right)$. The Bayes risks $R\left(\delta_{B 1}\right)=R\left(0,0,(0), 0, \delta_{B 1}\right)$ have been computed and tabulated in Table 2 . We see that $R\left(\delta_{B 1}\right)<R\left(\mathrm{SP}^{o}\right)$ for all cases. Thus, we conclude that the sampling plan $\left(0,0,(0), 0, \delta_{B 1}\right)$ outperforms the sampling $\operatorname{plan}\left(n^{o}, m^{o},\left(R_{1}^{o}, \ldots, R_{m^{o}}^{o}\right), T^{o}, \xi^{o}\right)$.

4.2. Comparison with Type-II APHC. Lin and Huang [3] have also studied the acceptance sampling with the loss $L$ and with type-II APHC using a type of decision function $\delta^{\mathrm{LH} 2}$, which is defined below. With the type-II APHC samples, the MLE of the expected lifetime $\theta$ is $\widetilde{\theta}_{2}=Y_{2}(n, m, K, T) / m$. The decision function $\delta^{\mathrm{LH} 2}$ is defined as

$$
\delta^{\mathrm{LH} 2}(K, \underline{Y}(m))= \begin{cases}1, & \text { if } \tilde{\theta}_{2} \geq \xi \\ 0, & \text { otherwise }\end{cases}
$$

A sampling plan with the type of decision functions is denoted by $\left(n, m,\left(R_{1}, \ldots, R_{m}\right), T, \xi\right) \quad$ (or by 
TABLE 3: Lin and Huang's [3] sampling plans and the associated MBR for type-II APHCS with $a_{0}=a_{1}=1, C_{s}=1, r_{s}=0.5$, and $C_{5}=$ $1-n^{o} / 1000$.

\begin{tabular}{|c|c|c|c|c|c|c|c|c|c|c|c|}
\hline Case & $(\alpha$ & $\beta$ & $a_{2}$ & $C_{t}$ & $\left.C_{r}\right)$ & $\left(n^{o}\right.$ & $m^{o}$ & $\left(\underline{R}^{o}\right)$ & $T^{o}$ & $\left.\xi^{o}\right)$ & MBR \\
\hline 1 & 1.5 & 2.0 & 1.0 & 2 & 5 & 4 & 1 & (3) & 18.0196 & 0.170 & 7.1844 \\
\hline 2 & 2.0 & 2.0 & 1.0 & 2 & 5 & 3 & 1 & (2) & 4.4317 & 0.1506 & 6.7917 \\
\hline 3 & 2.5 & 2.0 & 1.0 & 2 & 5 & 2 & 1 & (1) & 3.5540 & 0.4657 & 6.9126 \\
\hline 4 & 3.0 & 2.0 & 1.0 & 2 & 5 & 2 & 1 & (1) & 0.7569 & 0.7870 & 6.9878 \\
\hline 5 & 3.5 & 2.0 & 1.0 & 2 & 5 & 2 & 1 & (1) & 0.2151 & 1.1080 & 7.0326 \\
\hline 6 & 4.0 & 2.0 & 1.0 & 2 & 5 & 2 & 1 & (1) & 1.7355 & 1.4288 & 7.0347 \\
\hline 7 & 5.0 & 2.0 & 1.0 & 2 & 5 & 2 & 1 & (1) & 00674 & 2.0698 & 6.9722 \\
\hline 8 & 1.5 & 0.8 & 1.0 & 2 & 5 & 3 & 1 & (2) & 7.0134 & 1.0200 & 7.4685 \\
\hline 9 & 1.5 & 1.0 & 1.0 & 2 & 5 & 3 & 1 & (2) & 6.1897 & 0.8201 & 7.4974 \\
\hline 10 & 1.5 & 3.0 & 1.0 & 2 & 5 & 3 & 1 & (2) & 17.3696 & 0.0255 & 7.9389 \\
\hline 11 & 1.5 & 4.0 & 1.0 & 2 & 5 & 3 & 1 & (2) & 6.4334 & 0.0340 & 8.9738 \\
\hline 12 & 1.5 & 5.0 & 1.0 & 2 & 5 & 2 & 1 & (1) & 50.1664 & 0.0426 & 12.9870 \\
\hline 13 & 1.5 & 10.0 & 1.0 & 2 & 5 & 2 & 1 & (1) & 14.3119 & 0.0851 & 22.7314 \\
\hline 14 & 1.5 & 2.0 & 2.0 & 2 & 5 & 4 & 1 & (3) & 8.0285 & 0.4204 & 7.9139 \\
\hline 15 & 1.5 & 2.0 & 2.5 & 2 & 5 & 4 & 1 & (3) & 18.0813 & 0.6642 & 8.1161 \\
\hline 16 & 1.5 & 2.0 & 3.0 & 2 & 5 & 4 & 1 & (3) & 15.4680 & 0.8851 & 8.2648 \\
\hline 17 & 1.5 & 2.0 & 5.0 & 2 & 5 & 4 & 1 & (3) & 0.7582 & 1.6245 & 8.6104 \\
\hline 18 & 1.5 & 2.0 & 7.5 & 2 & 5 & 4 & 1 & (3) & 9.0591 & 2.3632 & 8.8203 \\
\hline 19 & 1.5 & 2.0 & 10.0 & 2 & 5 & 4 & 1 & (3) & 8.4516 & 2.9867 & 8.9406 \\
\hline 20 & 1.5 & 2.0 & 1.0 & 0 & 5 & 1 & 1 & $(0)$ & 15.0155 & 0.0170 & 3.6923 \\
\hline 21 & 1.5 & 2.0 & 1.0 & 1 & 5 & 3 & 1 & (2) & 6.9000 & 0.0170 & 6.0203 \\
\hline 22 & 1.5 & 2.0 & 1.0 & 3 & 5 & 5 & 1 & (4) & 4.0155 & 0.0170 & 8.0817 \\
\hline 23 & 1.5 & 2.0 & 1.0 & 4 & 5 & 5 & 1 & (4) & 18.5816 & 0.0170 & 8.8817 \\
\hline 24 & 1.5 & 2.0 & 1.0 & 5 & 5 & 5 & 1 & $(4)$ & 14.6429 & 0.0170 & 9.6817 \\
\hline 25 & 1.5 & 2.0 & 1.0 & 10 & 5 & 5 & 1 & $(4)$ & 16.9107 & 0.0170 & 13.6817 \\
\hline 26 & 1.5 & 2.0 & 1.0 & 2 & 10 & 4 & 1 & $(3)$ & 11.3484 & 0.0170 & 7.2475 \\
\hline 27 & 1.5 & 2.0 & 1.0 & 2 & 15 & 4 & 1 & $(3)$ & 4.5194 & 0.0170 & 7.3107 \\
\hline 28 & 1.5 & 2.0 & 1.0 & 2 & 20 & 4 & 1 & (3) & 5.9694 & 0.0170 & 7.3739 \\
\hline 29 & 1.5 & 2.0 & 1.0 & 2 & 30 & 4 & 1 & (3) & 1.0250 & 0.0170 & 7.5002 \\
\hline 30 & 1.5 & 2.0 & 1.0 & 2 & 50 & 4 & 1 & $(3)$ & 15.3002 & 0.0170 & 7.7528 \\
\hline 31 & 1.5 & 2.0 & 1.0 & 2 & 100 & 4 & 1 & (3) & 15.1794 & 0.0170 & 7.3844 \\
\hline
\end{tabular}

MBR: the Bayes risk reported in Lin and Huang [3].

$\left(\underline{R}^{o}\right)=\left(R_{1}^{o}, \ldots, R_{m}^{o}\right)$.

$\left.\left(n, m,\left(R_{1}, \ldots, R_{m}\right), T, \delta^{\mathrm{LH} 2}\right)\right)$. The Bayes risk of SP $=(n, m$, $\left.\left(R_{1}, \ldots, R_{m}\right), T, \xi\right)$ can be presented as

$$
\begin{aligned}
& R\left(n, m,\left(R_{1}, \ldots, R_{m}\right), T, \xi\right) \\
& \quad=S_{1}(\mathrm{SP})+S_{2}(\mathrm{SP})+S_{3}(\mathrm{SP})+S_{4}(\mathrm{SP})+S_{5}(\mathrm{SP})
\end{aligned}
$$

where

$$
\begin{aligned}
& S_{1}(\mathrm{SP})=C_{r} E\left[1-\delta^{\mathrm{LH} 2}(K, \underline{Y}(m))\right] \\
& S_{2}(\mathrm{SP})=\left(1-\frac{n}{N}\right) E\left[h(\lambda) \delta^{\mathrm{LH} 2}(K, \underline{Y}(m))\right] \\
& S_{3}(\mathrm{SP})=n\left(C_{s}-r_{s}\right), \\
& S_{4}(\mathrm{SP})=m r_{s}, \\
& S_{5}(\mathrm{SP})=C_{t} E\left[Y_{m: m, n}\right] .
\end{aligned}
$$

Lin and Huang [3] claimed they have derived the Bayesian sampling plans $\mathrm{SP}^{o}=\left(n^{o}, m^{o},\left(R_{1}^{o}, \ldots, R_{m^{o}}^{o}\right), T^{o}, \xi^{o}\right)$. The values of parameters of the sampling plans $\left(n^{o}, m^{o}\right.$, $\left.\left(R_{1}^{o}, \ldots, R_{m^{o}}^{o}\right), T^{o}, \xi^{o}\right)$ and the associated Bayes risks have been provided in Table 4 of Lin and Huang [3] (also, see Table 3 of the present paper). With the provided values of parameters of the sampling plans $\left(n^{o}, m^{o},\left(R_{1}^{o}, \ldots, R_{m^{o}}^{o}\right), T^{o}, \xi^{o}\right)$, the corresponding $S_{j}(\mathrm{SP})$ values, $j=1, \ldots, 5$, and the Bayes risks $R\left(\mathrm{SP}^{o}\right)$ are calculated and reported in Table 4 . We can verify that, for each sampling plan $\mathrm{SP}^{o}$ provided in Table 3 , the following inequality holds:

$$
\begin{aligned}
& S^{1,2}\left(\delta_{B 2} \mid n^{o}, n^{o},\left(n^{o} \times 0\right), 0\right)+n^{o}\left(C_{s}-r_{s}\right)+m^{o} r_{s} \\
& \quad+C_{t} E\left[X_{\left(m^{o}\right): n^{o}}\right]>\min \left(E h, C_{r}\right) .
\end{aligned}
$$

Thus, all the derived sampling plans $\left(n^{o}, m^{o},\left(R_{1}^{o}\right.\right.$, $\left.\left.\ldots, R_{m^{o}}^{o}\right), T^{o}, \xi^{o}\right)$ are not Bayesian sampling plans. 
TABLE 4: Comparison of performance of $\left(n_{B 2}, m_{B 2},\left(R_{1}^{*}, \ldots, R_{m_{B 2}}^{*}\right), T_{B 2}, \delta_{B 2}\right)$ and $\left(n^{o}, m^{o},\left(R_{1}^{o}, \ldots, R_{m^{o}}^{o}\right), T^{o}, \xi^{o}\right)$ for type-II APHCS sampling plans with $a_{0}=a_{1}=1, C_{s}=1, r_{s}=0.5$, and $C_{5}=1-n^{o} / 1000$.

\begin{tabular}{|c|c|c|c|c|c|c|c|c|}
\hline Case & $S_{1}$ & $S_{2}$ & $S_{3}$ & $S_{4}$ & $S_{5}$ & $R\left(\mathrm{SP}^{o}\right)$ & MBR & $R\left(\delta_{B}\right)$ \\
\hline 1 & 0.06308 & 2.62129 & 2.0 & 0.5 & 2.0000 & 7.1844 & 7.1844 & 2.6875 \\
\hline 2 & 0.67575 & 2.78271 & 1.5 & 0.5 & 1.3333 & 6.7917 & 6.7917 & 3.5000 \\
\hline 3 & 2.03725 & 2.04206 & 1.0 & 0.5 & 1.3333 & 6.9126 & 6.9126 & 4.4375 \\
\hline 4 & 3.15223 & 1.33561 & 1.0 & 0.5 & 1.0000 & 6.9878 & 6.9878 & 5.0000 \\
\hline 5 & 3.93121 & 0.80140 & 1.0 & 0.5 & 0.8000 & 7.0326 & 7.0326 & 5.0000 \\
\hline 6 & 4.42121 & 0.44683 & 1.0 & 0.5 & 0.6667 & 7.0347 & 7.0347 & 5.0000 \\
\hline 7 & 4.85670 & 0.11555 & 1.0 & 0.5 & 0.5000 & 6.9722 & 6.9722 & 5.0000 \\
\hline 8 & 3.54287 & 0.85895 & 1.5 & 0.5 & 1.0667 & 7.4685 & 7.4685 & 5.0000 \\
\hline 9 & 2.96377 & 1.20026 & 1.5 & 0.5 & 1.3333 & 7.4974 & 7.4974 & 5.0000 \\
\hline 10 & 0.06308 & 1.87578 & 1.5 & 0.5 & 4.0000 & 7.9389 & 7.9389 & 1.9167 \\
\hline 11 & 0.06308 & 1.57732 & 1.5 & 0.5 & 5.3333 & 8.9737 & 8.9738 & 1.6094 \\
\hline 12 & 0.06323 & 1.42382 & 1.0 & 0.5 & 10.0000 & 12.9870 & 12.9870 & 1.4500 \\
\hline 13 & 0.06315 & 1.16829 & 1.0 & 0.5 & 20.0000 & 22.7314 & 22.7314 & 1.1875 \\
\hline 14 & 1.24436 & 2.16953 & 2.0 & 0.5 & 2.0000 & 7.9139 & 7.9139 & 3.6250 \\
\hline 15 & 1.74789 & 1.86820 & 2.0 & 0.5 & 2.0000 & 8.1161 & 8.1161 & 4.0938 \\
\hline 16 & 2.11415 & 1.65069 & 2.0 & 0.5 & 2.0000 & 8.2648 & 8.2648 & 4.5625 \\
\hline 17 & 2.95052 & 1.15990 & 2.0 & 0.5 & 2.0000 & 8.6104 & 8.6104 & 5.0000 \\
\hline 18 & 3.44830 & 0.87201 & 2.0 & 0.5 & 2.0000 & 8.8203 & 8.8203 & 5.0000 \\
\hline 19 & 3.73003 & 0.71057 & 2.0 & 0.5 & 2.0000 & 8.9406 & 8.9406 & 5.0000 \\
\hline 20 & 0.06308 & 2.62918 & 0.5 & 0.5 & 0.0000 & 3.6923 & 3.6923 & 2.6875 \\
\hline 21 & 0.06308 & 2.62392 & 1.5 & 0.5 & 1.3333 & 6.0203 & 6.0203 & 2.6875 \\
\hline 22 & 0.06308 & 2.61866 & 2.5 & 0.5 & 2.4000 & 8.0817 & 8.0817 & 2.6875 \\
\hline 23 & 0.06308 & 2.61866 & 2.5 & 0.5 & 3.2000 & 8.8817 & 8.8817 & 2.6875 \\
\hline 24 & 0.06308 & 2.61866 & 2.5 & 0.5 & 4.0000 & 9.6817 & 9.6817 & 2.6875 \\
\hline 25 & 0.06308 & 2.61866 & 2.5 & 0.5 & 8.0000 & 13.6817 & 13.6817 & 2.6875 \\
\hline 26 & 0.12616 & 2.62129 & 2.0 & 0.5 & 2.0000 & 7.2474 & 7.2475 & 2.6875 \\
\hline 27 & 0.18924 & 2.62129 & 2.0 & 0.5 & 2.0000 & 7.3105 & 7.3107 & 2.6875 \\
\hline 28 & 0.25232 & 2.62129 & 2.0 & 0.5 & 2.0000 & 7.3736 & 7.3739 & 2.6875 \\
\hline 29 & 0.37848 & 2.62129 & 2.0 & 0.5 & 2.0000 & 7.4998 & 7.5002 & 2.6875 \\
\hline 30 & 0.63079 & 2.62129 & 2.0 & 0.5 & 2.0000 & 7.7521 & 7.7528 & 2.6875 \\
\hline 31 & 1.26159 & 2.62129 & 2.0 & 0.5 & 2.0000 & 8.3829 & 7.3844 & 2.6875 \\
\hline
\end{tabular}

$R\left(\delta_{B}\right)$ : the Bayes risk of the sampling plan $\left(n_{B}, m_{B},\left(R_{B 1}, \ldots, R_{B m_{B}}\right), T_{B}, \delta_{B}\right)$. $R\left(\mathrm{SP}^{o}\right)$ : the recalculated Bayes risk $R\left(n^{o}, m^{o},\left(R_{1}^{o}, \ldots, R_{m^{o}}^{o}\right), T^{o}, \xi^{o}\right)$.

MBR: the Bayes risk reported in Lin and Huang [3].

By applying Algorithm B, for each case studied in Table 3, we have derived the Bayesian sampling plan. It happens that, for all cases studied, the Bayesian sampling plans are $\left(0,0,(0), 0, \delta_{B 2}\right)$. The Bayes risks $R\left(0,0,(0), 0, \delta_{B 2}\right)=R\left(\delta_{B 2}\right)$ have been computed and tabulated in Table 4 . We see that $R\left(\delta_{B 2}\right)<R\left(\mathrm{SP}^{o}\right)$ for all cases. Thus, we conclude that the sampling plan $\left(0,0,(0), 0, \delta_{B 2}\right)$ outperforms the sampling plan $\left(n^{o}, m^{o},\left(R_{1}^{o}, \ldots, R_{m^{o}}^{o}\right), T^{o}, \xi^{o}\right)$.

\section{Concluding Remarks}

This paper deals with the acceptance sampling with the loss $L$ based on type-I APHC and type-II APHC samples. Algorithms are developed for searching the Bayesian sampling plans. We have compared the performance of the proposed sampling plans with sampling plans of Lin and Huang [3]. The numerical results indicate that the proposed sampling plans outperform the sampling plans of Lin and Huang [3].

Lin and Huang [3] have applied the same type of decision functions (see (55) and (61)) and similar approaches with type-I and type-II APHC samples for searching the Bayesian sampling plans. When we examine their approach carefully, we can see that there are certain deficiencies regarding the derivation of their sampling plans. First, note that the type of decision function $\delta^{\mathrm{LH} j}, j=1,2$, is based on the MLE $\widetilde{\text {. How- }}$ ever, with the adaptive progressive hybrid censored samples, $\widetilde{\theta}$ is not sufficient for the parameter $\theta$. Thus, the information contained in the samples regarding the parameter $\theta$ is not fully used when the decision functions $\delta^{\mathrm{LH} j}$ are applied. Secondly, we note that the decision function $\delta^{\mathrm{LH} j}$ is only an intuitive decision function, instead of being derived through the Bayesian analysis. Thus, $\delta^{\mathrm{LH} j}$ are not Bayesian decision 
functions. The performance of the sampling plans can be improved when a Bayesian decision function is applied.

Berger [4] has considered theoretic decision problem with no sample data. For all the 62 cases studied in Tables 1 and 2 , it happens that the derived Bayesian sampling plan is $\left(0,0,(0), 0, \delta_{B j}\right), j=1,2$, the sampling plan with no sampled data. The sampling plan $\left(0,0,(0), 0, \delta_{B j}\right)$ performs much better than the sampling plan $\left(n^{o}, m^{o},\left(R_{1}^{o}, \ldots, R_{m^{o}}^{o}\right), T^{o}, \xi^{o}\right)$ of Lin and Huang [3]. However, Lin and Huang [3] ignore making decisions with no sampled data.

\section{Conflict of Interests}

The author declares that there is no conflict of interests regarding the publication of this paper.

\section{References}

[1] J. Chen, W. Chou, H. Wu, and H. Zhou, "Designing acceptance sampling schemes for life testing with mixed censoring," Naval Research Logistics, vol. 51, no. 4, pp. 597-612, 2004.

[2] T. Liang and M.-C. Yang, "Optimal Bayesian sampling plans for exponential distributions based on hybrid censored samples," Journal of Statistical Computation and Simulation, vol. 83, no. 5, pp. 920-938, 2013.

[3] C.-T. Lin and Y.-L. Huang, "On progressive hybrid censored exponential distribution," Journal of Statistical Computation and Simulation, vol. 82, no. 5, pp. 689-709, 2012.

[4] J. O. Berger, Statistical Decision Theory and Bayesian Analysis, Springer, 1985. 


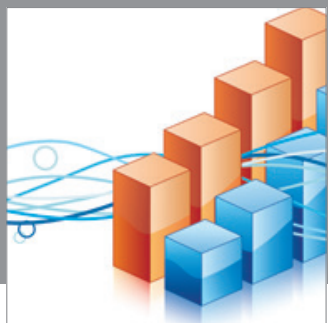

Advances in

Operations Research

mansans

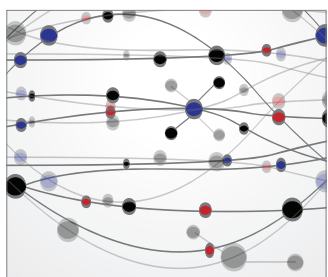

The Scientific World Journal
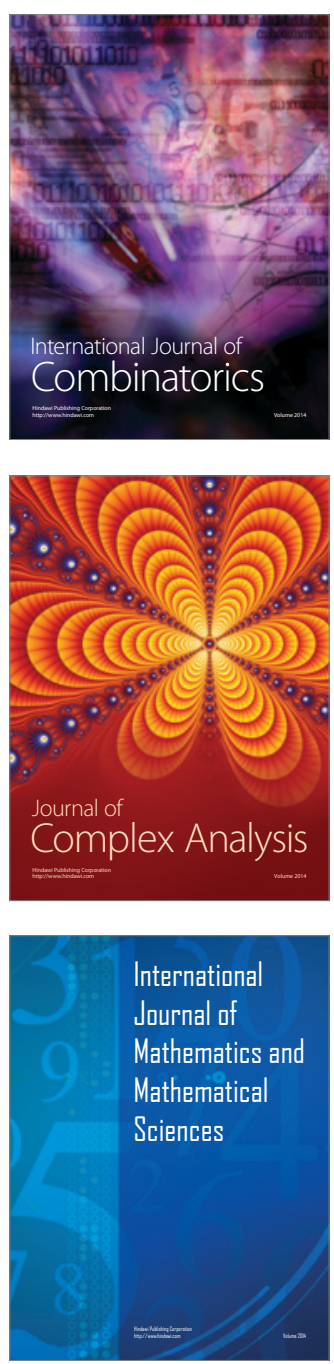
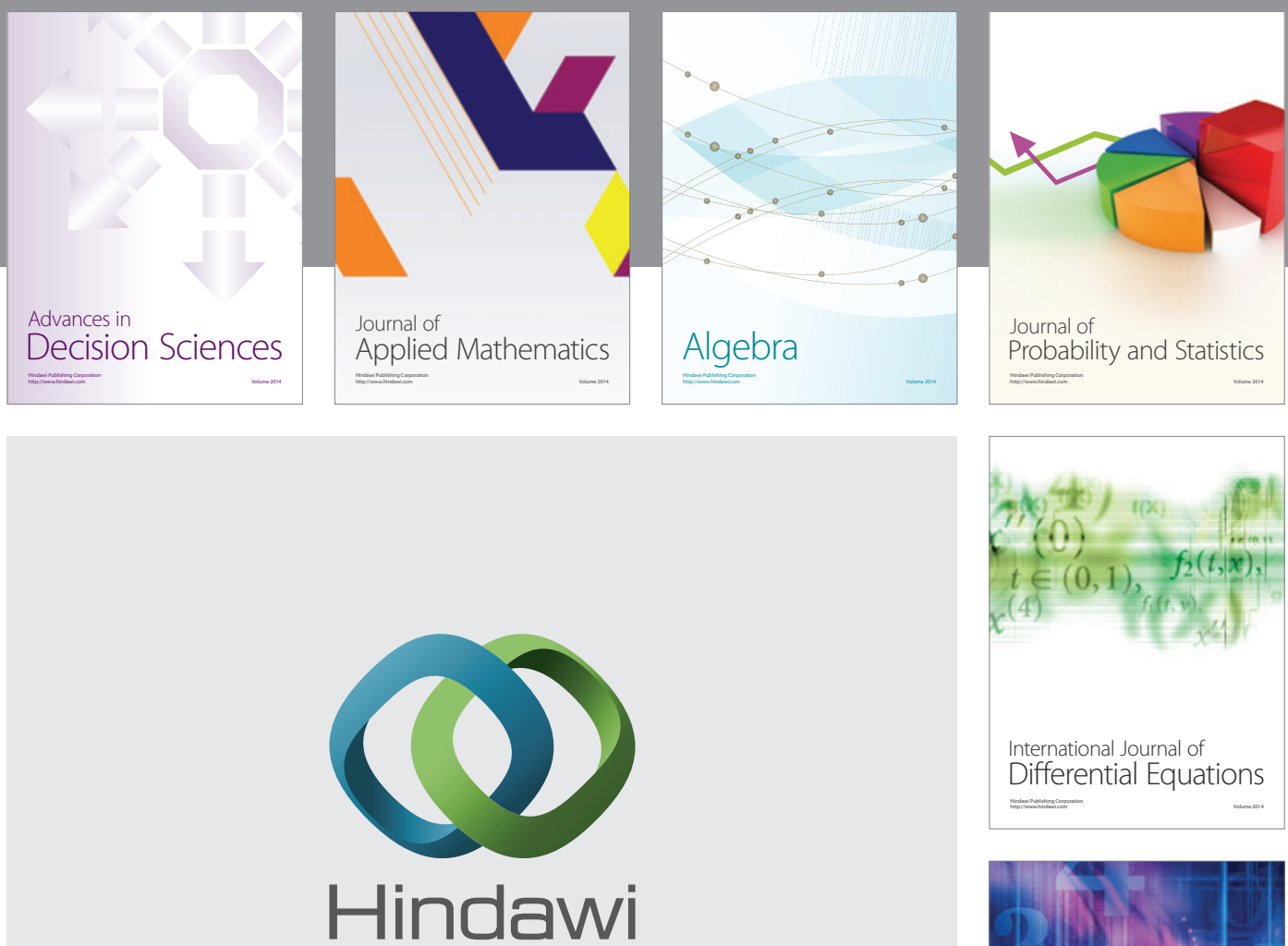

Submit your manuscripts at http://www.hindawi.com
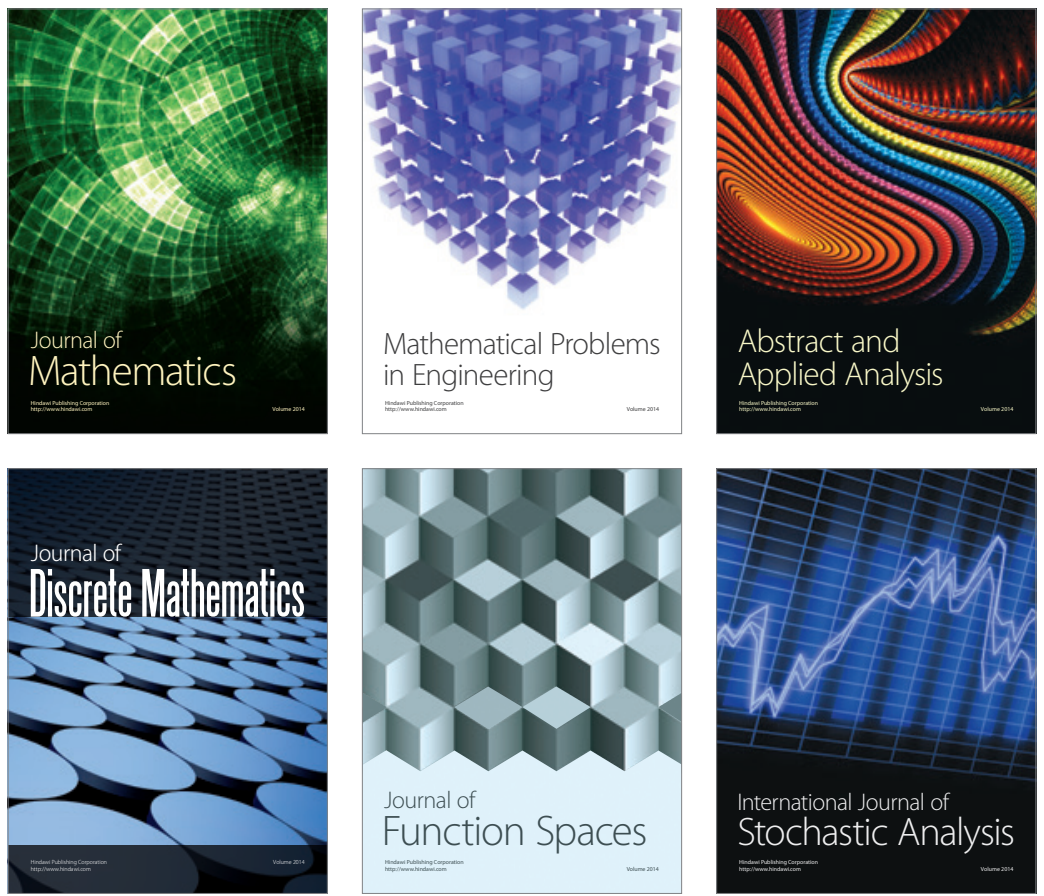

Journal of

Function Spaces

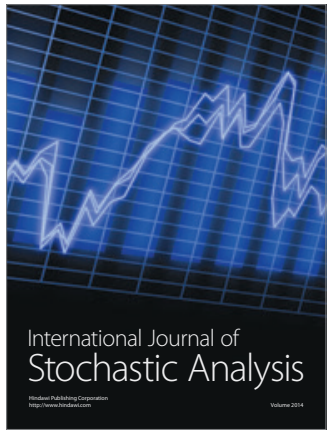

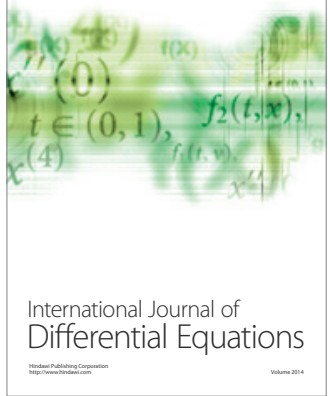
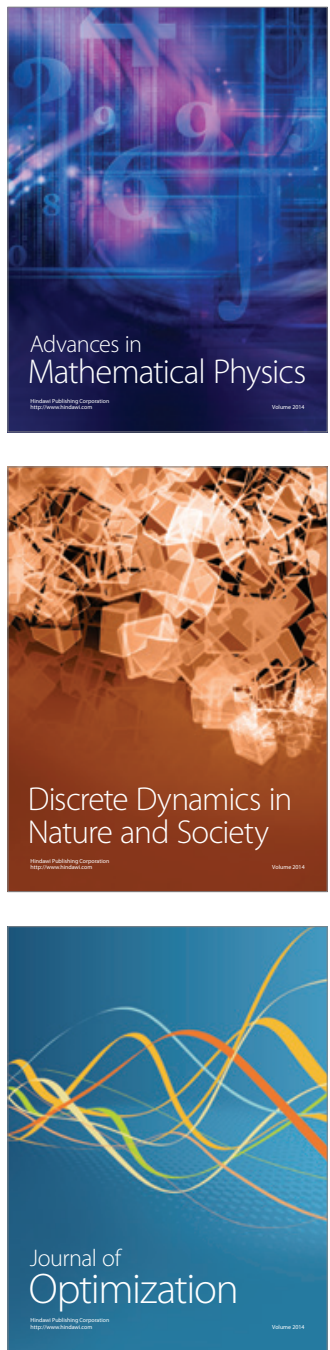